\title{
Activités
}

15-1| 2018

Mettre en scène l'activité : les nouvelles figures de la simulation

\section{Travail de nuit en 12 heures : un «scénario de travail » élaboré par les infirmiers dans un service de réanimation}

12-hour night shift: a "work scenario" developed by nurses in an intensive care unit

\section{Marlène Cheyrouze et Béatrice Barthe}

\section{OpenEdition}

\section{Journals}

Édition électronique

URL : https://journals.openedition.org/activites/3073

DOI : 10.4000/activites.3073

ISSN : 1765-2723

Éditeur

ARPACT - Association Recherches et Pratiques sur les ACTivités

Référence électronique

Marlène Cheyrouze et Béatrice Barthe, «Travail de nuit en 12 heures : un « scénario de travail » élaboré par les infirmiers dans un service de réanimation ", Activités [En ligne], 15-1 | 2018, mis en ligne le 15 avril 2018, consulté le 21 septembre 2021. URL : http://journals.openedition.org/activites/3073 ; DOI : https://doi.org/10.4000/activites.3073

Ce document a été généré automatiquement le 21 septembre 2021.

\section{(i) (3)}

Activités est mis à disposition selon les termes de la licence Creative Commons Attribution - Pas d'Utilisation Commerciale - Pas de Modification 4.0 International. 


\title{
Travail de nuit en 12 heures : un "scénario de travail » élaboré par les infirmiers dans un service de réanimation
}

\author{
12-hour night shift: a "work scenario" developed by nurses in an intensive \\ care unit
}

Marlène Cheyrouze et Béatrice Barthe

\section{NOTE DE L'ÉDITEUR}

Article soumis le 11/08/2017, accepté le 08/02/2018

Nous remercions les porteurs de ce projet de recherche, qui ont permis l'accès au terrain et défendu l'intérêt de l'étudier : Hervé Barrau (cadre infirmier du service), qui a été à l'initiative du projet, Marie-Hélène Requena, Sophie Bentz (membres de la Coordination de la Recherche) et Jean-Philippe Vauthier (Responsable scientifique de la Société Fédérative de la Recherche). Merci à tout le personnel du service de Réanimation Médicale pour leur accueil. Nous remercions tout particulièrement les infirmiers et les aides-soignants qui ont participé de près ou de loin à cette étude et qui nous ont accordé leur confiance en nous laissant toute latitude pour mener cette étude.

\section{Cadre de l'étude}

\subsection{Une étude initiée par une demande du terrain}

Ce travail de recherche s'inscrit dans un contexte d'essor des horaires 2 x 12 en milieu hospitalier. Il a été suscité par le cadre infirmier d'un service de réanimation qui fonctionnait en horaires $2 \times 12$ depuis 2009. Sa demande initiale était d'étudier l'impact 
de ces horaires sur la qualité de soins, et de repérer les stratégies employées par les soignants pour y faire face. Le Centre Hospitalier Universitaire s'est alors rapproché d'une équipe scientifique spécialiste de ces questions. Compte tenu de la grande diversité des tâches et des contextes entre le travail de jour et le travail de nuit, il a été décidé de focaliser l'étude sur le poste de nuit. Cette étude porte sur les liens entre le travail de nuit en 12 heures, la vigilance, la qualité des soins et les moyens de régulation mis en œuvre par les infirmiers dans leur activité de travail.

\subsection{Le travail en 12 heures : un succès grandissant en milieu hospitalier}

2 En France, le travail en 12 heures dans le secteur hospitalier remonte aux années 1980 et concernait au départ les infirmières spécialisées des blocs opératoires et services de réanimation (Vincent, 2014). Il se déploie aujourd'hui dans une grande diversité de services hospitaliers. En analysant les bilans sociaux de 348 établissements de santé, l'ATIH (2014) constate que deux tiers d'entre eux ont adopté les horaires 2 x 12 et que $10,5 \%$ d'agents hospitaliers sont concernés. Une enquête nationale sur les horaires en 12 heures a été menée en 2014 par l'Association pour le développement des ressources humaines dans les établissements sanitaires et sociaux (Girier, 2014). Parmi les 136 établissements répondants, plus de la moitié (51\%) comprennent moins de $10 \%$ des personnels soignants travaillant en 12 heures. Dans cette enquête, le personnel non médical concerné par l'organisation en 12 heures comprend principalement les infirmiers, les aides-soignants et les agents de services de sécurité incendie.

3 Le succès du travail en 12 heures est grandissant parce qu'il présente des intérêts à la fois pour les employeurs et pour les salariés. D'après des calculs de l'École des Hautes Études en Santé Publique, les horaires $2 \times 12$ permettraient à l'hôpital de réduire son effectif de $3,5 \%$ par rapport aux $7 \mathrm{~h} \mathrm{30/10} \mathrm{h} \mathrm{(} 7 \mathrm{~h} 30$ le matin; $7 \mathrm{~h} 30$ l'après-midi; $10 \mathrm{~h}$ la nuit) (TecHôpital.com, 2013). Les 12 heures auraient également des effets positifs sur le travail des soignants. Dans le cadre d'un module interprofessionnel de santé publique porté sur les horaires atypiques à l'hôpital, Brun et al. (2011) ont mené des entretiens ethnographiques auprès d'infirmiers et aides-soignants de 8 établissements publics et privés. Les témoignages sur le travail en 12 heures révèlent que le rallongement de la période de soin offre une meilleure connaissance des patients et permet de mieux les accompagner. Selon Vincent (2014), le travail en 12 heures offrirait la possibilité de « lisser » la charge de travail et de ne plus être stressé par le surcroît de travail, parfois inachevé et laissé aux collègues prenant la relève. Enfin, le rallongement de la durée journalière de travail à 12 heures entraine une diminution des jours travaillés. Vincent précise qu'il est en moyenne de 12 jours par mois, au lieu de 20 jours minimum avec des horaires en 8 heures. Le temps personnel libéré et la diminution des frais de transports induits par la diminution des jours travaillés sont des avantages énoncés dans plusieurs études sur les horaires $2 \times 12$ (Knauth, 2007). Ceci étant, la mise en place de ces horaires doit s'accompagner d'une prise en compte des risques identifiés sur le travail et les individus (Barthe, 2009). 


\subsection{Les effets du travail de nuit en 12 heures}

\subsubsection{Travail de nuit : les performances cognitives et le niveau de vigilance menacés}

4 L'étude présentée ici s'intéresse aux effets du poste de nuit de 12 heures sur le personnel infirmier. Selon Geiger-Brown (Geiger-Brown, \& al., 2010, p. A102-A103), le rôle de l'infirmier est de «maintenir une présence vigilante et de détecter des changements parfois subtils dans l'état des patients, afin de veiller aux complications». Or les performances cognitives sont régulées à la fois par le système circadien et par la pression de sommeil, et ceci de façon indépendante ou non (Anses, 2016). Selon le moment du cycle jour-nuit pendant lequel une performance cognitive est requise, le temps de réaction et la capacité d'attention soutenue, qui caractérisent le niveau de vigilance, seront différents. Dans le travail de nuit, la conjonction d'une heure biologique inappropriée et d'une veille de longue durée abaisse très sévèrement les performances cognitives et le niveau de vigilance. Ainsi, plusieurs études montrent que le travail posté incluant la nuit est associé à une baisse des performances cognitives (Anses, 2016). Celle de Ferguson, \& al. (2011) révèle que les performances des mineurs au PVT (Psychomotor Vigilance Task) sont plus altérées à la fin du poste de nuit qu'à la fin du poste de jour. Une autre étude de Geiger-Brown et al. (2012) portant sur 80 infirmières en 12 heures met en évidence que la vigilance - mesurée avec le PVT - est associée à la réduction sévère du temps de sommeil observée au cours des postes successifs et que la somnolence - mesurée avec la KSS (Karolinska Sleepiness Scale) - augmente plus vite durant le poste de nuit. Dans une autre étude de Cheeseman et al. (2011) le temps de réaction d'étudiants en anesthésie, lié à la reconnaissance d'étiquetage des médicaments sur ordinateur, est plus long lors des postes de nuit que lors des postes de jour. Enfin, l'étude de Machi et al. (2012) portant sur 13 médecins urgentistes montre que le poste de nuit a plus d'impact que le poste de jour sur la mémoire et certains processus cognitifs et d'apprentissage, ce qui peut avoir des conséquences lors d'une prise de décision en fin de poste de nuit. Selon Folkard et Åkerstedt (1992) c'est l'interaction entre privation de sommeil et durée de l'éveil qui explique les difficultés des travailleurs de nuit à maintenir un niveau de vigilance élevé tout au long du poste.

\subsubsection{Travail de nuit de 12 heures : des effets aggravés avec la durée d'éveil prolongée}

Lorsque le poste de nuit dure 12 heures, les effets du travail de nuit sur la vigilance sont renforcés via la durée de l'éveil. Brun et al. (2011, p.11) repèrent «un sentiment d'inquiétude quant aux risques d'erreurs" chez les professionnels de santé depuis l'instauration des 2 x 12 même si « aucune recrudescence d'événements indésirables n'a pu être observée ». Dans le milieu hospitalier, un événement indésirable correspond à « tout incident préjudiciable à un patient hospitalisé survenu lors de la réalisation d'un acte de prévention, d'une investigation ou d'un traitement " (Décret $n^{\circ}$ 2010-1408 du 12 novembre 2010). Plusieurs études montrent que le risque d'erreurs et d'accidents augmente au fil du poste. Pour déterminer l'existence d'un lien entre l'occurrence d'erreurs et les heures travaillées par les infirmiers, et identifier si ces heures travaillées ont des effets néfastes sur la vigilance des infirmiers, Scott, Rogers, Hwang et Zhang (2006) font remplir à ces derniers des journaux de bord. En se basant sur un échantillon 
de 502 répondants, ils montrent que plus la durée du poste est longue, plus le risque d'erreurs et de "presque erreurs » est important. Ici, le terme "presque erreur » fait référence à une erreur qui a été interceptée avant de se produire. De leur côté, Folkard et Tucker (2003) mettent en évidence une augmentation exponentielle du risque d'accidents de travail à partir de la neuvième heure travaillée. Le risque relevé dans les 8 premières heures est plus que doublé à la douzième. Dans leur étude menée sur des personnels de santé, Marias, Brillman et Tandberg (1996) montrent également que les accidents d'exposition biologique sont plus nombreux dans les deux dernières heures du poste en 12 heures. Les effets du travail de nuit sur la vigilance des soignants semblent aggravés par la durée de l'éveil, prolongée à 12 heures, et au-delà en tenant compte du temps de trajet pour rentrer au domicile. En étudiant les travailleurs d'une centrale nucléaire en poste de 12,5 heures, Heslegrave et al. (2000, in Knauth, 2007) montrent que ceux qui travaillent de nuit sont plus nombreux ( $41 \%$ contre $25 \%$ pour les travailleurs de jour) à s'endormir en conduisant sur le trajet du retour au domicile.

\subsection{Le travail hospitalier : un système dynamique aux exigences spécifiques la nuit}

\subsubsection{Les exigences du travail hospitalier la nuit}

6 Pour traiter la question des horaires, Barthe (2009) insiste sur l'importance de prendre en compte les caractéristiques du travail à accomplir: travail plutôt physique ou plutôt cognitif, travail actif ou de surveillance, travail isolé ou en équipe, etc. Or, le travail hospitalier de nuit s'inscrit dans un contexte bien particulier. Les médecins et le cadre de proximité sont absents du service et les soignants sont souvent moins nombreux que le jour (Toupin, 2008). La nuit, les besoins psychologiques et relationnels des patients sont forts (Manus, 2002). Les soignants sont seuls à y répondre, en plus d'avoir à gérer les incidents (désordres électriques, pannes diverses, manque de matériels, etc.) (Perraut Soliveres, 2002) et les situations difficiles (aggravation de l'état de santé d'un patient, agressions, etc.) pouvant survenir au cours de la nuit. Barthe (1999) identifie 3 types d'exigences potentiellement renforcées dans le travail hospitalier de nuit. Les effets de l'équipe étant réduits, les déplacements et les efforts de manutention sont plus nombreux: cela renvoie aux exigences physiques. La baisse nocturne de la vigilance entraine des difficultés de traitement de l'information et de concentration : cela renvoie aux exigences cognitives. Enfin, la mort d'un patient prend une dimension plus angoissante la nuit, ce qui renvoie aux exigences psychologiques.

\subsubsection{Le travail hospitalier : un système dynamique}

7 Le travail hospitalier s'inscrit dans un système dynamique. Selon Hoc (2004) les «situations dynamiques » évoluent sous le contrôle partiel de l'opérateur humain. Elles sont déterminées par: 1) l'étendue du champ de supervision et de contrôle; 2) la proximité du contrôle ; 3) la vitesse du processus et 4) un certain degré d'incertitude et de risque. Lancry (2016) propose la notion «environnement dynamique» pour mieux comprendre les conditions de certains travailleurs. Selon lui, le travail hospitalier s'inscrit dans cet environnement dynamique, qui présente des caractéristiques similaires à celles décrites par Hoc. La première caractéristique est l'incertitude qui, en milieu hospitalier, est liée au nombre et à l'état des patients à prendre en charge, et qui évoluent 
en permanence. La deuxième caractéristique est le contrôle distant, puisque l'effet de certains soins et traitements n'est connu que plusieurs heures, voire plusieurs jours après l'intervention du professionnel de santé. La troisième caractéristique est le risque lié à la nature et à l'importance des actes. Le degré de contrôle et la vitesse du processus dépendent de l'état des patients, qui lui-même va définir les priorités et l'importance des actes à prodiguer. Selon Gonon, Barthe et Gindro (2009), le travail hospitalier est influencé par plusieurs temporalités: celle du soignant (fatigue, vigilance...), celle du patient (évolution de l'état physiologique et psychologique), celle de l'équipe soignante, celle des autres professionnels du service (médecin, cadre infirmier...) et celle des autres services (radiologie, laboratoire, blanchisserie, hôtellerie...). À partir d'exemples d'interventions, ils montrent comment ces différents niveaux de temporalités peuvent "s'entrechoquer" ou "s'articuler» avec la temporalité des soignants et celle des patients. Le caractère imprévisible du travail des personnels hospitaliers réside dans la rencontre de toutes ces temporalités, mais aussi dans l'évolution de l'état (physique, psychique et psychiatrique) des patients. Le temps consacré à un patient peut largement différer d'un jour à l'autre ou d'un patient à l'autre. Ainsi, l'imprévisibilité du travail des personnels hospitaliers peut être liée d'une part à la dégradation non prévue de l'état d'un patient et d'autre part aux dysfonctionnements dans les transferts d'informations interprofessionnels et/ou interservices.

\subsection{Le travail hospitalier : un système maîtrisé par la régulation de l'activité}

8 Comme dans toute situation de travail et pour tout opérateur, c'est dans leur activité de travail que les soignants font face aux effets du travail de nuit en 12 heures, l'activité pouvant être définie comme « une stratégie d'adaptation à la situation réelle de travail » (Guérin, Laville, Daniellou, Duraffourg, \& Kerguelen, 1997, p. 34). Cette activité ne correspond pas à l'application des consignes prescrites, mais à un processus de régulation qui permet aux travailleurs d'atteindre leurs objectifs par « la gestion des variations tant du système technico-organisationnel que de leur propre état interne " (Noulin, 2002, p. 39). Nous considérons que les processus de régulation employés par les soignants sont pluriels et qu'ils peuvent leur permettre simultanément de gérer leur baisse de vigilance et atteindre leurs objectifs de soin au cours du poste de nuit de 12 heures.

\subsubsection{La régulation de l'activité pour maintenir l'équilibre du système}

Dans le modèle de Leplat (2000) l'opérateur régule son activité pour: A) éviter les répercussions potentiellement négatives de l'activité sur lui-même; B) atteindre les objectifs de la tâche; C) gagner en compétences. L'activité s'assimile donc à un compromis entre les "conditions internes" liées à l'opérateur (âge, état de santé, expérience, fatigue, stress, vigilance, etc.) et les " conditions externes » liées à la tâche (buts, niveaux d'exigence, moyens, etc.). Par ailleurs, cette activité a des effets sur l'opérateur en termes de santé (somnolence, douleurs, accident, etc.) et de compétences (apprentissage), et sur la tâche en termes de performance. En fonction de l'écart constaté entre son état initial et l'état produit par son activité, et de la performance visée à la performance effective, l'opérateur régule son activité pour rétablir l'équilibre du système. Falzon (2004) précise que la régulation peut se faire en «boucle longue » en évaluant les résultats a posteriori ou en « boucle courte » en évaluant a priori l'évolution du 
système et en agissant avant le relevé d'un écart. Selon Gadbois et Quéinnec (1984) l'activité mise en place la nuit est déterminée par les variations circadiennes des capacités psychophysiologiques des opérateurs. Ce lien est lui-même modulé par les exigences de la tâche.

\subsubsection{Un « scénario de travail » construit sur l'anticipation des variations de la charge de travail et de la vigilance}

10 Le travail du soignant s'inscrit bien dans un système dynamique, et s'assimile à du contrôle de processus. Nous retenons que ce processus est marqué par l'imprévisibilité, la situation de travail du soignant pouvant évoluer en dehors de toute action de sa part. En effet, l'état des patients évolue de façon continue, sans être nécessairement lié à l'intervention du soignant. Par ailleurs, plusieurs acteurs gravitent autour d'un même patient: leurs interventions plus ou moins directes peuvent se heurter à celles du soignant et avoir des effets non prévus par ce dernier sur la situation de travail. À cela s'ajoute les aléas logistiques (manque de matériel, appareil qui ne fonctionne pas, lit manquant, etc.) et administratifs (dossier médical incomplet, erreur d'identité, etc.). Selon Van Daele et Carpinelli (2001, p. 4), le risque de perdre le contrôle du processus est au centre des préoccupations de l'opérateur. De façon permanente, celui-ci vise les objectifs suivants: "être dans le temps du processus", "ne pas se faire déborder", « avoir une marge de manœuvre » et " être en avance plutôt qu'en retard ». Pour éviter de perdre le contrôle du processus, il cherche à évaluer a priori l'évolution du système et agir avant le relevé d'un écart entre les entrées et les sorties (Falzon, 2004). L'anticipation est le socle de la maitrise du processus. Selon Le Bris, Tirilly et Toupin $(2015$, p. 3$)$ les stratégies d'anticipation s'affirment avec l'expérience et visent deux objectifs pour la nuit de travail : limiter la fatigue et éviter les urgences pour avoir « une activité plus sereine et si possible bien maitrisée». Pour faire face aux pics de fatigue (faible vigilance) et d'activité (gestion d'une entrée, aggravation de l'état d'un patient, quantité importante de nouvelles prescriptions médicales, etc.), il devient nécessaire pour les opérateurs d'anticiper les variations de la charge de travail et de leur état psychophysiologique. Barthe (2015) fait une synthèse de plusieurs stratégies identifiées dans la littérature, ayant pour objectif «d'anticiper et de se réserver des marges de manœuvre pour faire face au mieux aux événements imprévus qui pourraient survenir au cours des heures » (p. 5). Ces stratégies sont relevées au début du poste de nuit et s'assimilent à une prise d'informations exhaustive sur la situation de travail et à un regroupement des tâches les plus exigeantes mentalement. De notre point de vue, les opérateurs se construisent un «scénario de travail » qui se caractérise par une planification de l'activité et par une actualisation régulière de la situation de travail.

11 S'il veut maitriser le processus, l'activité de l'opérateur doit satisfaire à de fortes exigences de planification (Van Daele, \& Carpinelli, 2001). La planification vise à maintenir l'évaluation du processus dans des limites acceptables. Elle est définie comme «l'élaboration et/ou l'utilisation de représentations schématiques et/ou hiérarchisées (plans) susceptibles de guider l'activité » (p. 2). L'anticipation est un aspect essentiel de la planification puisqu'elle consiste à produire une représentation qui précède l'exécution : cette représentation s'assimile selon nous à un «scénario de travail». Van Daele et Carpinelli distinguent deux moments de planification: quand l'opérateur prépare l'exécution (il élabore son scénario) et quand l'opérateur est déjà dans l'exécution (il modifie son scénario en fonction des imprévus). Pour reprendre les concepts de Falzon 
(2004) nous considérons qu'en préparant l'exécution, l'opérateur est dans la régulation en «boucle courte » (évaluer a priori l'évolution du système) et qu'en étant dans l'exécution il est dans la régulation en «boucle longue » (évaluer a posteriori). Ainsi, le mécanisme de production d'un plan (d'un scénario) consiste à déterminer les contraintes à prendre en compte, à sélectionner d'éventuelles solutions partiellement connues ou à élaborer de nouvelles solutions, et à affiner et mettre en œuvre progressivement ces solutions.

12 À son arrivée sur le poste, l'opérateur cherche à savoir ce qui s'est produit en son absence et dans quel état se trouve le processus : il actualise ainsi sa représentation mentale (Barthe, Quéinnec, \& Verdier, 2004). En 1996, Andorre et Quéinnec ont montré que les contrôleurs de processus prélevaient deux fois plus d'informations au début de poste que lors de la tenue de poste. Cette prise d'informations globale d'environ 90 minutes leur permet d'actualiser leur représentation du système et de disposer de connaissances pour anticiper les variations futures. Toupin (2008) identifie ce processus d'actualisation à l'hôpital, dans un service de pneumologie où les infirmières de nuit font un premier tour $\mathrm{du}$ service pour visiter leurs patients avant qu'ils ne s'endorment. Cette première visite leur permet de se représenter la nuit qu'elles vont passer. Dans un autre service de réanimation, Cheyrouze, Barthe et Barrau (2016) montrent que c'est tout au long du poste que les soignants réactualisent leur représentation de la situation de travail, en avançant un maximum de tâches : l'étiquetage des bilans sanguins, la préparation des seringues, le rechargement des réserves de matériel ou les constantes. Prendre de l'avance leur permet d'avoir une vision précise de la charge de travail à venir et de se rendre disponibles pour gérer les urgences potentielles et l'augmentation de leur somnolence.

\subsubsection{Un scénario de travail déroulé sur le reste du poste pour gérer la baisse de vigilance}

13 Dans la littérature, Barthe (2015) identifie d'autres stratégies relevées au moment du pic de fatigue : le transfert de tâches non prioritaires, la diminution des déplacements et des communications, la modification du mode opératoire de certaines tâches et l'aménagement de temps de repos. Plusieurs études montrent que l'activité s'accorde à la rythmicité circadienne de la vigilance en atteignant un minimum entre $1 \mathrm{~h}$ et $3 \mathrm{~h}$ du matin; il peut s'agir de l'activité de surveillance (de Terssac, Quéinnec, \& Thon, 1983), l'activité locomotrice (déplacements), ou l'activité verbale (échanges verbaux) (Christol, Dorel, Quéinnec, \& de Terssac, 1979). Dans l'étude de Toupin (2008, p. 226), les infirmières de pneumologie regroupent les phases de rédaction des transmissions entre $0 \mathrm{~h}$ et $2 \mathrm{~h}$, période où elles se sentent encore suffisamment alertes et éveillées, puis les complètent en fin de nuit. Si cela est possible et non préjudiciable pour l'état de santé du patient, certaines infirmières cherchent à avancer la préparation et l'exécution de plusieurs soins du milieu de nuit $(4 \mathrm{~h})$, par exemple un antibiotique. Cette organisation leur permet d'éviter les erreurs éventuelles dues à la fatigue et que ces soins n'empiètent sur leurs «moments de tranquillité nocturnes » qui leur permettent de «se poser et se reposer ». Le mode opératoire de la tâche peut aussi changer en période de faible vigilance: Barthe (1999) montre dans un service de néonatologie que pour les bébés en alimentation mixte (sonde stomacale ou tétée) sur 24 heures, les soignantes privilégient l'alimentation par sonde stomacale qui est 5 fois plus rapide que l'alimentation au biberon. Elle montre que si la santé de l'enfant le permet, elles peuvent éviter certaines tâches jugées secondaires telles que le changement de couche ou les soins de bouche et d'ombilic. Ces réaménagements leur permettent non seulement de préserver le sommeil des enfants, 
mais aussi d'écourter leur durée de mobilisation entre $2 \mathrm{~h}$ et $5 \mathrm{~h}$, période sur laquelle elles prennent du repos avant la dernière phase de soins qui est la plus difficile physiquement et mentalement.

Les stratégies de régulation que nous venons de décrire témoignent d'une anticipation de l'état de vigilance futur et permettent d'aménager des temps de repos et/ou de sieste. Si les soignantes du service de néonatologie écourtent les soins de $2 \mathrm{~h}$ du matin, c'est pour gagner du temps de repos et de micro sommeil avant les soins de $5 \mathrm{~h}$ et être capable de "tenir " jusqu'à la fin du poste (Barthe, \& Quéinnec, 2005). Geiger-Brown et Trinkoff (2010) recommandent une sieste de 20 minutes dans un lieu calme pour une nuit de travail en 12 heures. Ses effets sont bénéfiques sur les fonctions cognitives (Matsumoto, \& Harada, 1994 ; Bonnefond, 2002) et la performance (Smith, \& Wilson, 1990). Barthe, Tirilly, Gentil et Toupin (2016) montrent notamment que les prises de repos spontanées améliorent le niveau de somnolence et la qualité de travail perçus par les infirmiers à la fin du poste de nuit en 10 heures.

\subsection{La qualité des soins : une notion multidimensionnelle et des objectifs portés par les soignants}

Cette étude s'intéresse à l'activité mise en œuvre par les infirmiers pour tenir les objectifs de travail tout au long d'un poste de nuit de 12 heures et viser un niveau optimal de qualité des soins. Cette dernière prend différentes dimensions selon le point de vue adopté. Dès 1987, l'OMS (Organisation Mondiale de la Santé) définit la qualité des soins comme « une démarche qui doit permettre de garantir à chaque patient l'assortiment d'actes diagnostiques et thérapeutiques qui lui assurera le meilleur résultat en termes de santé, conformément à l'état actuel de la science, au meilleur coût pour le même résultat, au moindre risque iatrogénique, pour sa plus grande satisfaction en termes de procédures, résultats, contacts humains à l'intérieur du système de soins. » (Parent, 2008, p. 9). Cette première définition montre la double exigence technique (thérapeutique) et humaine (contact avec le patient) de la qualité. En 2001, l'IOM (Institute of Medecine) définit la qualité des soins comme le fait de recevoir des soins qui correspondent aux attentes des patients et basés sur la meilleure connaissance scientifique actuelle. Nous retrouvons la dimension humaine caractérisée par la satisfaction du patient et la dimension technique reposant sur la connaissance. Nous faisons le parallèle avec la distinction faite en sciences sociales entre les notions de cure, qui relève du traitement thérapeutique de la maladie et de care, qui relève de l'accompagnement du patient dans l'exécution de ses gestes quotidiens (Saillant, 1999, in Lalandre, 2009). Or et Com-Ruelle (2008, p. 373) considèrent que la qualité des soins est devenue une «notion multidimensionnelle» et proposent cinq indicateurs pour la mesurer: l'efficacité (amélioration des résultats sanitaires), la sécurité (empêcher ou éviter les résultats indésirables issus des processus de soins), la réactivité (respect des patients, dignité, confidentialité, participation aux choix, soutien social), l'accessibilité (facilité d'accès aux soins hospitaliers) et l'efficience (utilisation optimale des ressources disponibles à moindres frais et en obtenant les meilleurs résultats). Tirilly, Barthe et Gentil (2015) soulignent que l'activité réelle du travail de soin et son contexte sont souvent mis à l'écart pour évaluer la qualité des soins. En poste de nuit par exemple, les soignants réinterprètent le travail à faire en se fixant des objectifs spécifiques. En service de pneumologie, Toupin et Volkoff (2007) identifient les objectifs que visent les infirmières 
et comment ils peuvent entrer en contradiction dans l'activité de soins : assurer la conformité et la qualité des soins, gérer l'angoisse des patients et protéger leur sommeil, gérer leur propre fatigue et leur propre somnolence, renforcer la connaissance de l'état des patients et favoriser la dimension collective du travail. Ces dimensions nous paraissent compatibles avec la notion du care, qui comporte selon Molinier (2006, in Gaudart, \& Thébault, 2012, p. 244) « des savoirs discrets qui mobilisent tout à la fois des connaissances techniques, le souci du confort psychologique de l'autre et l'économie de soi ». Car la qualité ne vient pas uniquement du prescrit: elle est aussi façonnée par l'activité réelle des travailleurs. La qualité ne se résume donc pas à la définition de normes et à la conformité du travailleur au prescrit. Il y a en réalité une articulation implicite entre le « prescrit » et le « réel » du travail, autrement dit une qualité construite par l'opérateur. La « qualité réglée » se distingue de la « qualité gérée » (Falzon, Dicioccio, Mollo, \& Nascimento, 2013). La première repose sur la formulation de règles (procédures, référentiels, prescriptions, etc.) et la volonté de s'assurer de leur application. La deuxième repose sur les capacités d'initiative des opérateurs, seuls ou en groupe, à faire face à l'imprévisibilité et la variabilité naturelle du réel. La qualité gérée consiste donc à juger la pertinence de l'application de la qualité réglée selon les circonstances et à mettre en œuvre un arbitrage raisonné permettant d'atteindre le meilleur niveau de qualité possible. Cet arbitrage peut fortement s'éloigner du prescrit, au point d'être transgressif. Mais face à l'imprévisibilité du travail, l'intervention humaine, et donc la régulation de l'activité par l'opérateur, est indispensable pour assurer la fiabilité, la qualité et la performance (Falzon \& al., 2013). Il est donc nécessaire d'étudier la qualité du point de vue de l'opérateur.

\subsection{Objectifs de l'étude}

Dans cette étude, nous choisissons d'étudier la qualité des soins à travers la perception du soignant, qui est détenteur de savoirs non reconnus dans le prescrit, et qu'il nous parait important de mettre en lumière. La qualité est façonnée par l'activité réelle des travailleurs; nous nous intéresserons alors aux objectifs spécifiques que se fixent les infirmiers en poste de nuit de 12 heures. Nous intégrerons dans notre analyse la notion du care, qui permet à l'infirmier de mobiliser à la fois ses connaissances techniques, sa volonté d'assurer le confort physique et psychologique du patient, et de gérer ses propres capacités psychophysiologiques. Dans la continuité des travaux déjà menés, les objectifs de cette étude sont de mettre en lien les stratégies d'anticipation et de planification des infirmiers que nous associons à un "scénario de travail » avec leur définition et leur perception subjectives de la qualité des soins, tout en prenant compte des variations de leur vigilance.

\section{Situation de travail étudiée}

\subsection{Le service de réanimation médicale}

Cette étude a été demandée par le cadre infirmier d'un service de réanimation médicale, pouvant accueillir en continu jusqu'à 20 patients. Il s'agit de patients en détresse vitale qui présentent une ou plusieurs défaillances viscérales aiguës (respiratoire, cardiovasculaire, rénale, hématologique, hépatique et neurologique) et/ou de grands 
brûlés. Les soignants assurent le fonctionnement des organes défaillants via des systèmes médicotechniques rigoureux. La prise en charge des patients exige une surveillance constante, des compétences techniques, des soins précis et une grande réactivité. Le personnel compte 55 infirmiers, 35 aides-soignants, 2 aides-soignantes techniques, 21 médecins (dont 10 internes), 2 kinésithérapeutes et 2 cadres infirmiers. Les équipes soignantes se relaient toutes les 12 heures à $7 \mathrm{~h}$ et $19 \mathrm{~h}$. Le temps de transmission est d'environ 15 minutes. Le planning est organisé sur un cycle de 5 semaines et induit théoriquement 14 jours/nuits travaillé(e)s, 11 repos compensateurs, 10 congés et 3 weekends libres. Au cours du cycle, les soignants travaillent 1 fois une journée/nuit de 12 heures suivie d'une période de repos, 5 fois deux journées/nuits consécutives et 1 fois trois journées/nuits consécutives. Pour combler l'effectif manquant dans l'équipe de nuit, les soignants de l'équipe de jour doivent travailler de nuit sur 3 cycles, soit environ 4 mois par an. Il y a donc deux types de soignants en poste de nuit : des soignants fixes de nuit et des soignants de jour ponctuellement de nuit. Le service est organisé en 4 modules de 5 lits chacun. Le premier module accueille les patients infectieux sévères, le deuxième les grands brûlés, le troisième les patients d'hématologie et le quatrième est polyvalent. Le service est conçu pour limiter les infections : les modules sont fermés et insonorisés; il est impossible de circuler de l'un à l'autre sans passer par le couloir central. La communication entre les modules et les différentes zones s'effectue par interphone. Il y a 2 infirmiers et un aide-soignant par module. À cela s'ajoutent un infirmier et un aidesoignant responsables de l'accueil en urgence des patients grands brûlés. Ces soignants apportent leur aide dans les modules selon la charge de travail. Sur un poste en 12 heures de jour et de nuit, il y a donc 9 infirmiers et 5 aides-soignants.

\subsection{La population étudiée}

18 Cette étude s'intéresse aux infirmiers de nuit, fixes et ponctuels. Les infirmiers qui ont participé sont tous volontaires et ont donné leur autorisation pour être suivis, observés et interrogés dans leur travail.

\subsection{Les tâches prescrites des infirmiers de nuit}

Tout au long du poste, le rôle de l'infirmier est de planifier, préparer et exécuter les soins prescrits par les médecins et d'assurer - en collaboration avec l'aide-soignant - les soins de confort quotidiens. Il doit pouvoir combiner sa compétence technique à une prise en charge attentive qui évalue les besoins, le confort et la douleur (physique/psychologique) du patient. Ses tâches prescrites sont illustrées dans la figure chronologique ci-dessous (Figure 1). 
Figure 1. Tâches des infirmiers sur un poste de nuit de 12 heures.

Figure 1. Nurses' tasks over the 12-hour shift

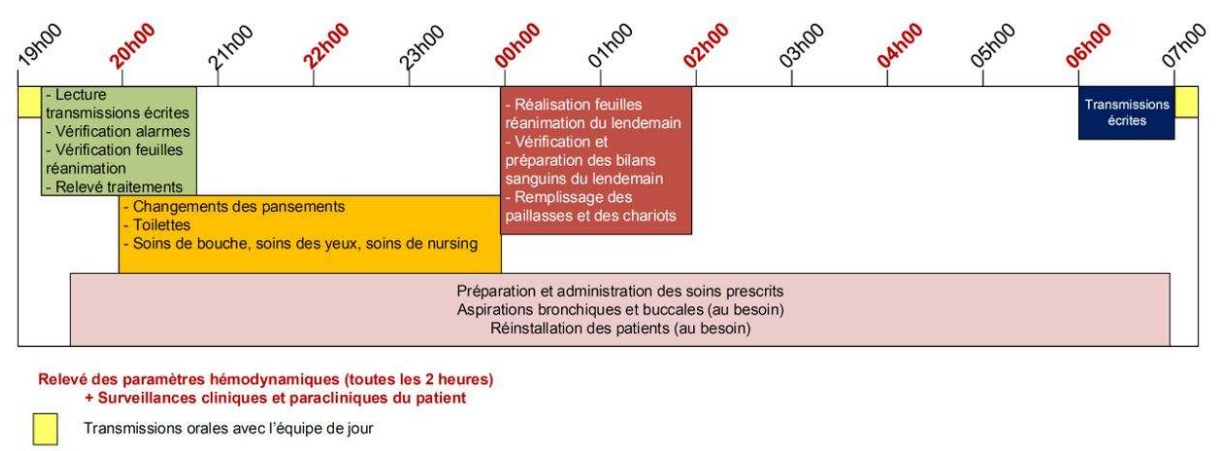

Après les transmissions orales avec l'équipe de jour, les infirmiers de nuit doivent procéder à une vérification des transmissions écrites (dossiers des patients), des prescriptions et du plan de soins (feuilles de réanimation), du patient (installation, état général) et de son environnement (fonctionnement des dispositifs techniques et des alarmes). En parallèle des traitements, les soins administrés entre $20 \mathrm{~h}$ et $00 \mathrm{~h}$ visent à " préparer le patient pour la nuit », autrement dit le mettre dans des conditions propices à son endormissement (propreté du patient et de son environnement, installation dans le lit, extinction des lumières, réduction des entrées dans la chambre). Certains soins sont à renouveler dans la nuit selon les prescriptions. Toutes les deux heures $(20 \mathrm{~h} ; 22 \mathrm{~h} ; 00 \mathrm{~h}$; $2 \mathrm{~h} ; 4 \mathrm{~h}$ et $6 \mathrm{~h}$ ), les infirmiers font «les tours »: ils relèvent les paramètres hémodynamiques (température, rythme cardiaque, tension, etc.) des patients, administrent les traitements prévus et renouvèlent si besoin certains soins de confort. Ils assurent tout au long du poste la surveillance clinique et paraclinique des patients en contrôlant régulièrement le scope médical, leur état physique, l'environnement technique, etc.

\subsection{Les exigences du travail infirmier en réanimation}

21 Les infirmiers ont la responsabilité des traitements et des soins techniques, particulièrement nombreux et complexes en service de réanimation. En effet, ils doivent maitriser plusieurs dispositifs techniques tels que le pousse-seringues, la pompe à perfusion, le défibrillateur cardiaque, le respirateur, l'appareil à dialyse, le scope médical, etc. Comme l'expliquent les opérateurs, la préparation et l'administration des traitements nécessitent un effort de concentration important (calculs de dosage, nombreux produits) et une dextérité précise (pansements complexes, aspirations trachéales, soins de bouche, etc.) Les infirmiers assurent la surveillance continue du patient, en se basant sur les systèmes de monitorage (fréquence cardiaque, pression artérielle, saturation en oxygène, fréquence respiratoire, température) et sur l'évaluation clinique (aspect et comportement du patient). Ces exigences témoignent du fait que les infirmiers doivent faire preuve d'anticipation et de réactivité pour faire face à tout moment aux situations d'urgences (arrêt cardiaque, désaturation, montée de température, extubation volontaire, etc.). 


\section{Méthodologie}

Cette étude a été menée sur une période de 8 mois, guidée par une méthodologie en 3 temps: un temps d'immersion (Phase 1), un temps d'exploration soutenue (Phase 2) et un temps de recueil systématique des données (Phase 3).

\subsection{Participants concernés pour chaque phase}

Le temps d'immersion (Phase 1) a porté sur 2 postes de nuit et 18 infirmiers. Le temps d'exploration soutenue (Phase 2) a concerné 12 infirmiers. Le temps de recueil systématique (Phase 3 ) a porté sur 9 postes de nuit pour un échantillon de 24 infirmiers (dont 6 ayant participé à la Phase 2). L'échantillon est composé de 3 hommes et 21 femmes. La moyenne d'âge est de 31,5 ans. L'ancienneté dans le métier d'infirmier est en moyenne de 8,2 ans et l'expérience de la réanimation est en moyenne de 6 ans. Parmi les 24 participants, 9 infirmiers sont de nuit toute l'année (fixes) et 15 infirmiers sont de nuit 4 mois par an (ponctuels).

\subsection{Phase 1 : Immersion dans le service}

Les objectifs de cette phase sont de se familiariser au service (rencontre avec le personnel, exploration des locaux), d'en comprendre l'organisation et d'appréhender le travail des infirmiers de nuit. En référence à nos objectifs, plusieurs variables font l'objet d'un premier ciblage : la somnolence et la vigilance, la qualité des soins et les stratégies de régulation. Une démarche d'observation, soutenue par l'utilisation d'un carnet de notes, est menée sur 2 postes de nuit auprès de 18 infirmiers. Les données recueillies font l'objet d'une analyse qualitative et sont catégorisées dans le compte-rendu ci-dessous.

\section{Données recueillies en phase d'immersion (Phase 1) \\ - Premiers constats}

- Le travail se réalise dans 4 modules fermés et insonorisés ce qui entraine : pas de visibilité instantanée sur la charge de travail du module voisin ; pas de communications spontanées; coopérations inter modules limitées; déséquilibres de la charge de travail d'un module à l'autre.

- Les infirmiers s'avancent en permanence pour : 1) Anticiper les imprévus potentiels ou la fatigue de fin de poste ; 2) Ne jamais se laisser dépasser (selon leurs témoignages).

- Manque de personnel fixe de nuit (compensé par les soignants de jour contraints de travailler de nuit 4 mois par an).

\section{- Particularités du poste de nuit}

Service beaucoup plus calme : les infirmiers sont seuls avec les aides-soignants et les médecins de garde ce qui leur permet de moduler plus librement leur organisation de travail ; la $1{ }^{\text {ère }}$ partie de la nuit est consacrée aux principales tâches 
(pansements, traitements, nursings) et la $2^{\text {de }}$ est consacrée à la surveillance, au relevé des constantes et au repos de l'équipe soignante.

Temps de repos : si la charge de travail le permet, les infirmiers s'installent dans des fauteuils médicaux jambes étendues et en position allongée. Certains ferment les yeux et disent être en " demi-sommeil ». Ce temps de repos est toléré par le cadre, qui se dit conscient des effets du travail de nuit sur la somnolence et convaincu des bienfaits des temps de repos et de « micro-sieste ».

Cette démarche d'immersion nous met face aux difficultés méthodologiques qui se posent pour l'étude. Le service de réanimation est immense (une superficie de $2000 \mathrm{~m}^{2}$ ) et les infirmiers sont dispersés dans quatre modules fermés et insonorisés. Il est impossible de tous les observer au même moment et la circulation entre les modules est contraignante car elle engage de nombreux déplacements, très coûteux en énergie. Observer les infirmiers d'un seul module induit un petit échantillon (deux infirmiers par module) et suivre un seul infirmier dans le cadre d'une observation systématique le réduit davantage. Pour obtenir un échantillon plus conséquent sur un poste de nuit en 12 heures, nous avons choisi de déployer dans la suite de l'étude une observation globale dans deux modules voisins.

\subsection{Phase 2 : Entretiens exploratoires avec les infirmiers}

Puisque nous n'employons pas l'observation systématique et que l'angle de vue de l'opérateur nous intéresse particulièrement, cette deuxième phase consiste à étudier nos variables (somnolence et vigilance, qualité des soins, stratégies de régulation) sous l'angle de la perception des infirmiers pour en définir les indicateurs, et créer des outils de mesure adaptés pour la phase suivante du recueil de données. Les variables sont introduites dans une grille d'entretien, élaborée avec les informations du terrain et de la littérature scientifique. L'entretien se divise en 5 parties et se compose de 17 questions (Annexe 1). La passation concerne 12 infirmiers de nuit. Il y a 1 homme et 11 femmes. La moyenne d'âge est de 31 ans. L'ancienneté moyenne dans le métier est de 7 ans et l'expérience moyenne de la réanimation est de 6 ans. Les données recueillies font l'objet d'une analyse qualitative et sont catégorisées dans le compte-rendu ci-dessous.

\section{Données recueillies en phase d'entretiens exploratoires (Phase 2)}

\section{- L'augmentation de la somnolence au cours des 12 heures : effets et stratégies associées}

Les infirmiers citent plusieurs effets de l'augmentation de la somnolence : 1) Un ralentissement de la cadence de travail lié à une perte d'énergie et de réactivité ; 2) Une perte de patience vis-à-vis des patients ou des collègues (plus d'agacement, moins d'écoute et de motivation) ; 3) Une baisse de vigilance s'accompagnant d'un plus gros effort de concentration. Plusieurs infirmiers savent à quelle période $\mathrm{du}$ poste ils vont se sentir plus somnolents : à partir de $2 \mathrm{~h}$, entre $4 \mathrm{~h}$ et $6 \mathrm{~h}$ ou entre $6 \mathrm{~h}$ et $7 \mathrm{~h}$. Pour éviter une éventuelle incidence sur leur travail, ils anticipent cette période en avançant un maximum de tâches. Au moment où la somnolence augmente, d'autres stratégies sont indiquées : 1) Chercher à s'occuper ; 2) Sortir de la zone de soins (prendre l'air, marcher, boire un café ou fumer une cigarette) ; 
3) Prendre un temps de repos (s'asseoir, poser la tête, s'allonger, fermer les yeux, somnoler).

\section{— La qualité du travail définie par les infirmiers}

Les infirmiers renvoient la qualité du travail à une prise en charge " globale », c'està-dire adaptée à chaque patient et effectuée dans le respect de ses besoins et des protocoles. La qualité de la prise en charge dépend de conditions extrinsèques et intrinsèques. Les conditions extrinsèques sont : un matériel fonctionnel ; un personnel soignant en effectif suffisant et en bonne forme physique et mentale ; de bonnes relations interprofessionnelles ; des locaux adaptés ; des délais suffisants ; un bon encadrement. Les conditions intrinsèques sont liées au soignant, qui doit : fournir un travail efficace ; être organisé ; prendre le temps de réfléchir et d'agir ; être à l'écoute de son patient ; « cadrer » ses patients en faisant les choses en temps et en heure sans se laisser dépasser ; être sérieux, rigoureux et au clair avec ce qu'il a à faire. La qualité du travail est évaluée à travers la satisfaction des patients et de son entourage, et à travers celle des soignants. La satisfaction de l'infirmier se traduit notamment par un sentiment d'avoir bien travaillé en quittant le service, un sentiment de bien-être et d'épanouissement personnel.

À un niveau plus opérationnel, les infirmiers définissent le " travail de qualité » selon 8 grands critères : 1) Le travail est accompli en temps et en heure sans s'être laissé débordé et sans avoir laissé de tâches au collègue de jour ; 2) Le service laissé à la relève est " cadré » avec des patients propres, confortablement installés, traités et apaisés, et avec un matériel complet et fonctionnel ; 3) La prise en charge du patient est « globale » ce qui signifie qu'on a respecté les conditions d'hygiène, accompagné les familles, expliqué les soins au patient, écouté le ressenti et les besoins du patient, installé confortablement le patient et limité sa douleur ; 4) Le patient fait confiance à l'infirmier et/ou le remercie ; 5) L'entente, la communication et la satisfaction sont favorables dans l'équipe ; 6) L'infirmier comprend son travail, des prescriptions jusqu'aux traitements ; 7) L'infirmier a une organisation correcte de ses soins et de son espace de travail, est efficace dans sa prise en charge et anticipe les tâches futures ; 8) L'infirmier respecte toutes les règles techniques/théoriques de l'hôpital.

\section{— Les situations " problématiques"}

Les critères du « travail bien fait » sont plus difficilement atteignables dans les situations suivantes : 1) Les urgences (nouvelle entrée, patient qui se dégrade); 2) Les patients agités (remuants, agressifs, bruyants) ; 3) Les surcharges de travail (dégradation simultanée de plusieurs patients, sollicitations multiples des collègues, personnel en sous-effectif, fortes exigences des médecins) ; 4) La mauvaise entente dans l'équipe (vision différente des méthodes et des objectifs, manque d'entraide) ; 5) Le surplus d'intervenants et d'interlocuteurs sur un seul patient ; 6) Le manque de matériel ; 7) La fatigue des soignants ; 8) Une mauvaise organisation.

\section{- Les stratégies pour faire face aux situations problématiques}

En situation «problématique » les infirmiers emploient 6 types de stratégie :

1) Prioriser selon l'urgence vitale, la douleur, le prescripteur de la tâche ;2) Déléguer à un autre soignant ; 3) Prendre sur soi en repoussant, raccourcissant ou diminuant 
ses pauses, voire en quittant plus tard le service ; 4) Faire sa propre planification du travail ; 5) Accélérer le rythme de son activité ; 6) Communiquer avec les médecins pour réduire leurs exigences ou soulager les patients, avec l'aide-soignant pour redéfinir l'importance des tâches.

\section{Les stratégies pour réduire le risque d'oublis et d'incidents}

Pour limiter les oublis et les incidents, les infirmiers emploient 6 types de stratégie :

1) La vérification multiple et répétée des feuilles de réanimation ;2) Le marquage visuel sur les feuilles de réanimation ; 3) La tenue d'un support écrit personnel ; 4) La préparation anticipée des soins ; 5) L'organisation mentale ; 6) Le travail d'équipe (communication, partage des tâches, rappel des tâches).

À partir de cette analyse qualitative, nous retenons les critères suivants pour étudier la qualité des soins du point de vue de l'infirmier :

- Le travail est accompli en temps et en heure sans s'être laissé débordé ;

- Les règles d'hygiène, les règles techniques et théoriques sont respectées ;

- Le matériel est complet et fonctionnel ;

- La prise en charge des patients est globale (adaptée à chaque patient et effectuée dans le respect de ses besoins et des protocoles);

- L'entente, la communication et la satisfaction sont favorables dans l'équipe ;

- L'infirmier comprend son travail, des prescriptions jusqu'aux traitements ;

- L'infirmier a une organisation correcte de ses soins et de son espace de travail, est efficace dans sa prise en charge, est au clair avec ce qu'il a à faire ;

- L'infirmier anticipe les tâches futures ;

- L'infirmier prend le temps de réfléchir et d'agir.

\section{planification suivantes :}

- Accélérer le rythme de son activité ;

- Prioriser selon l'urgence vitale, la douleur, le prescripteur de la tâche ;

- Anticiper la préparation des soins ;

- Vérifier plusieurs fois les feuilles de réanimation ;

- Tenir un support écrit personnel ;

- S'organiser mentalement ;

- Déléguer à un autre soignant ;

- Prendre sur soi en repoussant, raccourcissant ou diminuant ses pauses.

- Pour les périodes de faible vigilance, nous retenons les stratégies suivantes :

- Chercher à s'occuper ;

- Sortir de la zone de soins (prendre l'air, marcher, boire un café ou fumer une cigarette) ;

- Prendre un temps de repos (s'asseoir, poser la tête, s'allonger, fermer les yeux, somnoler).

\subsection{Phase 3 : Recueil de données systématiques}

Suite à l'analyse des entretiens exploratoires, les objectifs de cette dernière phase sont d'étudier les variables définitivement choisies au cours du poste en 12 heures: la 
vigilance, la qualité des soins et les stratégies de régulation. Cette $3^{\text {ème }}$ phase se caractérise par un couplage de l'analyse quantitative et de l'analyse qualitative.

\subsubsection{Relevé de l'activité : la technique de l'observation globale déployée tout au long du poste...}

Pour étudier l'activité de travail, plusieurs méthodes s'offrent à l'ergonome: l'observation, les verbalisations (quand l'activité est commentée par l'opérateur) et les mesures (de variables physiologiques par exemple). L'observation est presque toujours utilisée conjointement aux autres méthodes. Elle peut être un support de verbalisations ou produire des critères pour interpréter les mesures (Kerguelen, 1995). En ergonomie, il existe deux techniques d'observations (Simonet, Caroly, \& Clot, 2011; Barthe, Boccara, Delgoulet, Gaillard, Meylan, \& Zara-Meylan, 2017): l'observation systématique et l'observation globale (ou ouverte). Dans une précédente étude (Cheyrouze, Barthe, \& Barrau, 2016), nous avions choisi l'observation systématique en étudiant les stratégies de régulation mises en œuvre par les soignants au moyen d'une grille d'observation. Mais cet outil a présenté plusieurs limites. D'abord, il n'était pas exhaustif (certaines stratégies n'étaient pas répertoriées dans la grille). Ensuite, il était impossible d'avoir une visibilité constante sur l'ensemble des soignants (l'un dans une chambre, l'autre en salle de repos, l'autre dans la zone de soins, etc.) Dans le service de cette étude, le manque de visibilité lié à la sectorisation des quatre modules nous conduit à employer l'observation globale, soutenue par un carnet de notes et menée dans deux modules voisins. Cette technique a été employée dans la phase d'immersion (Phase 1) et se renouvelle dans le recueil de données (Phase 3) pour s'associer à d'autres techniques. L'observation globale permet d'appréhender les situations de travail sans avoir a priori défini ce qui serait à observer (Barthe, \& al., 2017). Dans notre cas, nous avions décidé de noter le plus d'informations possibles sur les infirmiers (actions, comportements) et sur la situation de travail (contexte, événements) en précisant l'heure et en passant d'un module à l'autre régulièrement (rester dans le même module 20 minutes au maximum). Barthe et al. (2017) expliquent que l'activité étant un processus en partie inobservable, l'analyse de l'activité ne peut bien entendu se limiter à l'observation. Dans cette étude, la technique de l'observation globale est le support de deux autres techniques déployées au cours du poste en 12 heures : le questionnaire et l'entretien.

\subsection{2. ... couplée à la passation de trois questionnaires et d'un entretien}

Dans l'approche quantitative cette étude, nous faisons le choix d'étudier les effets du poste de nuit en 12 heures au moyen de questionnaires. Comme le précisent PrunierPoulmaire et Gadbois (2005), l'information recueillie par un questionnaire ne fournit pas des faits mais la représentation que les opérateurs se font de la situation. La subjectivité de l'opérateur n'est pas problématique dès lors que sa représentation de la situation est un élément pertinent, et parfois critique. Cette représentation nous intéresse tout particulièrement. Notre démarche est similaire à celle de Prunier-Poulmaire et Gadbois (2005) qui ont étudié les conditions de travail des agents de brigades de Surveillance des Douanes en utilisant le questionnaire. Celui-ci est construit dans une seconde phase, après avoir mené des observations in situ ainsi que des entretiens individuels auprès des travailleurs concernés. Cette première phase d'exploration, que nous avons nous-même menée dans notre étude (Phase 2), permet d'obtenir une certaine connaissance du terrain 
et de l'activité. Elle confère au questionnaire toute sa pertinence et en fait un outil susceptible de refléter le plus fidèlement possible la situation de travail et les préoccupations des opérateurs. Pour ce qui est de la qualité des soins, nous considérons qu'elle est façonnée par l'activité réelle des opérateurs et qu'elle doit donc être étudiée avec la perception de ces derniers. En nous inspirant de la littérature et des entretiens exploratoires (Phase 2), nous avons donc créé une échelle d'auto-évaluation de la qualité des soins. Enfin, les processus de régulation mis en œuvre par les infirmiers sont indiqués par ces derniers au moyen d'un questionnaire, également inspiré de la littérature et des entretiens exploratoires.

L'infirmier remplit ces trois questionnaires, 4 fois au cours du poste. Il est interrogé après chaque passation sur le contenu de ses réponses en entretien individuel. La chercheuse est présente tout au long du poste dans le service et relève en continu des données d'observation. Le déroulé chronologique de cette méthodologie est illustré ci-dessous (Figure 2).

Figure 2. Organisation chronologique du recueil de données sur un poste de nuit en 12 heures. Figure 2. Chronological organisation of data collection over the 12-hour night shift

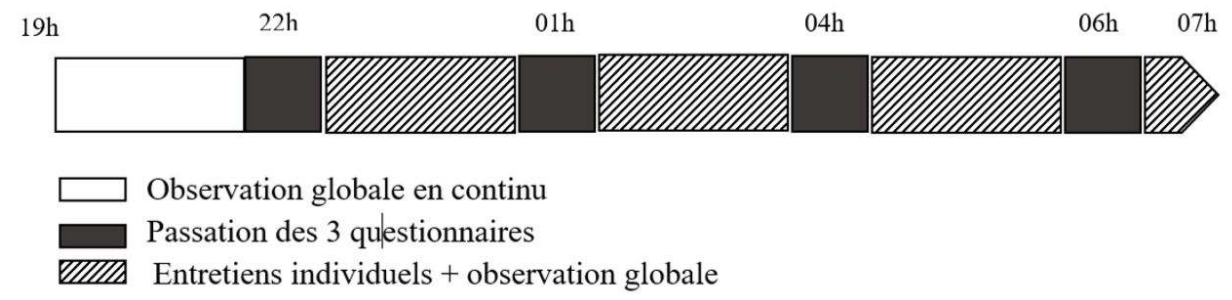

\subsubsection{Echelle d'auto-évaluation de la vigilance des soignants}

Pour mesurer la vigilance au cours du poste, l'échelle d'auto estimation «ActivationDeactivation Adjective Checklist » (Thayer, 1967) est complétée par le participant au bout de 3 heures travaillées, 6 heures, 9 heures et 11 heures (Annexe 2). 20 adjectifs sont proposés au participant : Actif (ve); Indifférent(e); Endormi(e); Mal à l'aise ; Energique ; Calme ; Fatigué(e); Dynamique; Détendu(e); Attentif (ve); Somnolent(e); Inquiet(e); «La pêche »; Tranquille ; Eveillé(e) ; Enervé(e) ; Silencieux (se); En forme ; Tendu(e) ; Crispé (e).

35 À chaque adjectif, le participant associe l'une des 4 modalités suivantes : je me sens très (4), je me sens un peu (3), je ne sais pas (2), je ne me sens pas du tout (1). Les adjectifs renvoient à 4 sous-échelles : l'énergie (Activation Générale - GA), la fatigue (Tendance au sommeil - DS), la tension (Hyperactivation - HA) et le calme (Hypo activation - GD). Le rapport GA/DS permet d'obtenir un indice de vigilance dont le seuil est égal à 2. Un indice inférieur à ce seuil s'apparente à une vigilance faible et un indice supérieur à une vigilance élevée. Les données obtenues ont été analysées avec les logiciels SPSS et Excel et ont fait l'objet d'un test statistique (test de Friedman).

\subsubsection{Echelle d'auto-évaluation de la qualité des soins par les soignants}

Pour mesurer la qualité des soins au cours du poste, une échelle d'auto-évaluation est complétée par le participant, seul, et aux mêmes temps que le questionnaire précédent (Annexe 3). Cette échelle comporte 11 critères de qualité spécifiques au service et à la 
population. Ces critères sont côtés « $Q$ ». Ils sont tirés de l'analyse qualitative des entretiens exploratoires (Phase 2) et de la littérature (cf. paragraphe 8.3), et sont formulés sous forme de questions. Le participant évalue chaque critère en choisissant parmi 4 couleurs : vert (positif), jaune (moyennement positif), orange (moyennement négatif) et rouge (négatif). Le score peut aller de 1 à 4 points. Plus l'auto-évaluation est positive, plus le score est élevé. Les données obtenues ont été analysées avec les logiciels SPSS et Excel et ont fait l'objet d'un test statistique (test de Friedman). Le tableau cidessous montre comment chaque critère du questionnaire (Phase 3) est lié aux entretiens exploratoires (Phase 2) et/ou à la littérature.

Tableau 1. Informations recueillies dans les entretiens exploratoires et la littérature et présentation des 11 critères du questionnaire d'auto-évaluation de la qualité des soins qui y sont associés. Table 1. Information gathered from the exploratory interviews and the literature, and presentation of the self-assessment questionnaire's 11 criteria relating to the quality of treatment.

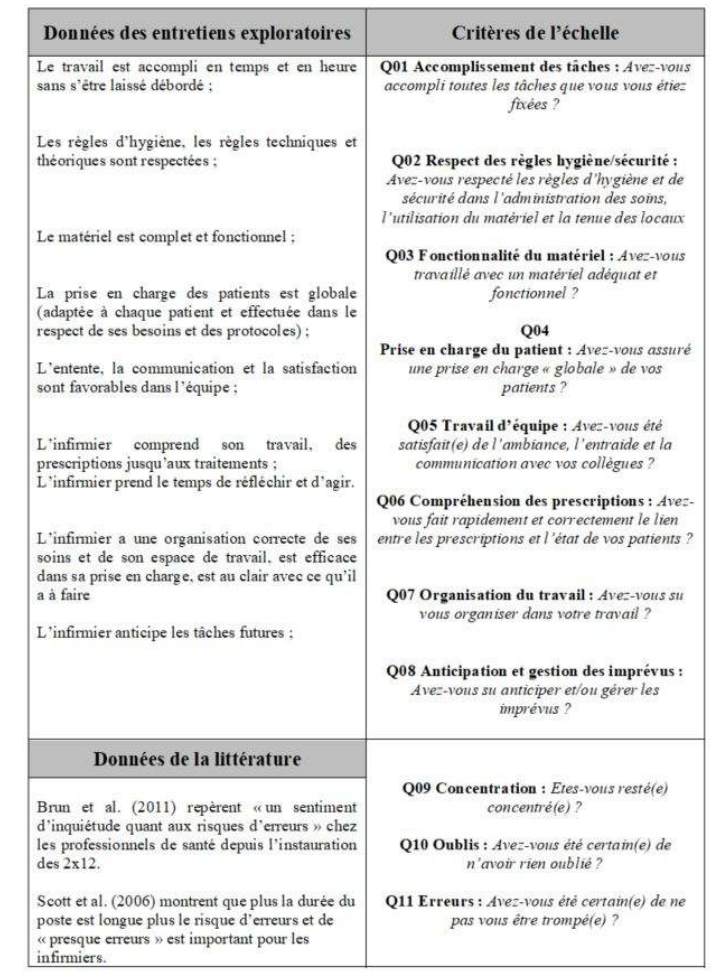

\subsubsection{Questionnaire : Repérage des stratégies de régulation}

Pour relever les stratégies de régulation des infirmiers au cours du poste, une liste de 9 stratégies (cotées « $\mathrm{S} »)$ est proposée au participant aux mêmes temps que les questionnaires précédents (Annexe 4). Ces stratégies sont tirées de l'analyse qualitative des entretiens exploratoires (Phase 2) et de la littérature (cf. paragraphe 8.3). Seul, le participant coche toutes celles qu'il a utilisées dans les dernières heures. Une stratégie utilisée est égale à 1 et une stratégie non utilisée égale à 0 . Un score d'utilisation est alors obtenu pour chaque stratégie. Les données obtenues ont été analysées avec les logiciels SPSS et Excel et ont fait l'objet d'un test statistique (test de Friedman). Le tableau cidessous montre comment chaque stratégie du questionnaire (Phase 3) est liée aux entretiens exploratoires (Phase 2). 
Tableau 2. Informations recueillies dans les entretiens exploratoires et présentation des 12 stratégies de régulation qui y sont associés dans le dernier questionnaire. Table 2. Presentation of the 12 regulation strategies linked, in the final questionnaire, to the information gathered from the exploratory interviews.

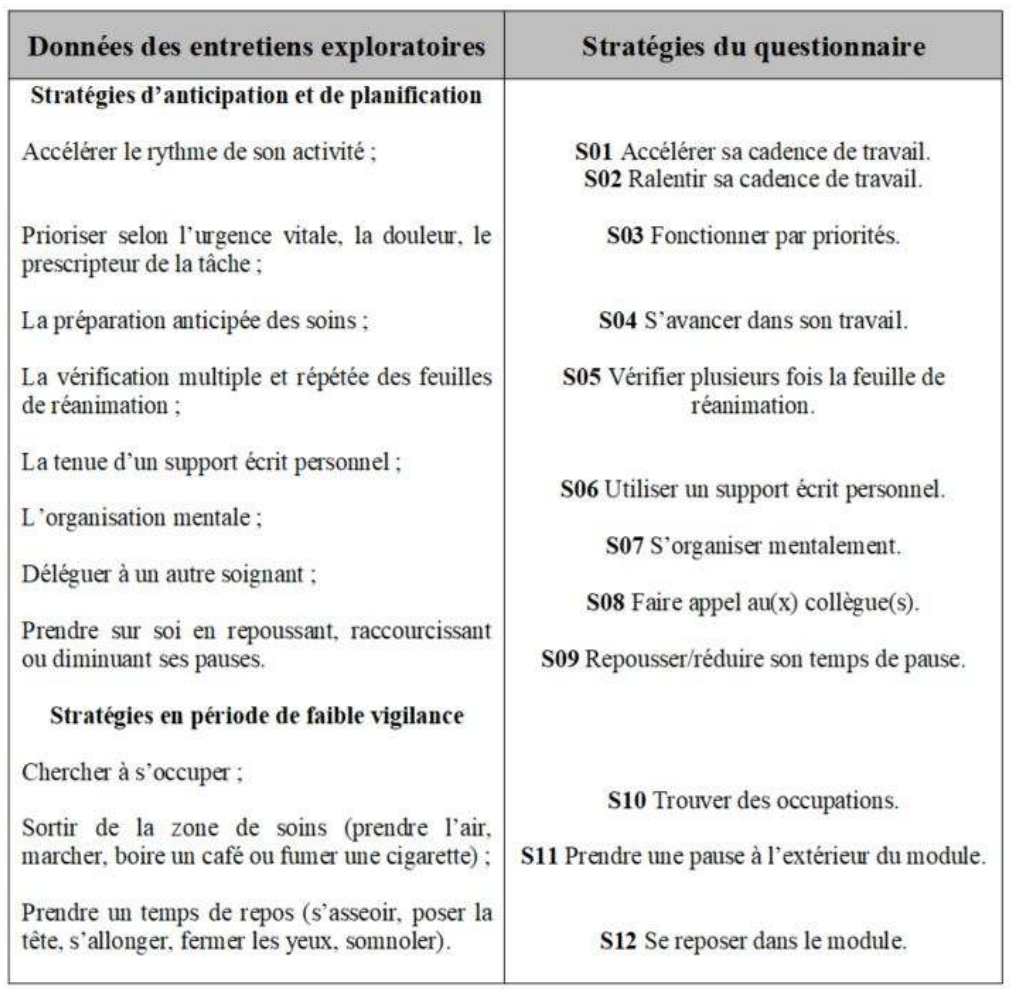

\subsubsection{Entretiens individuels après chaque passation des trois questionnaires}

Après la passation des questionnaires, des entretiens individuels sont menés auprès des participants. L'infirmier est invité à commenter son activité passée et vécue en s'appuyant sur ses réponses aux trois questionnaires. Ces entretiens s'inspirent à la fois de l'entretien d'explicitation et de l'entretien d'auto-confrontation. L'entretien d'explicitation est une technique d'aide à la verbalisation développée par Vermersch (1991) dont l'utilisation se situe a posteriori de l'activité qui fait l'objet de l'explicitation. L'objectif est de guider le sujet dans la verbalisation précise de ses actions passées. Dans l'entretien d'auto-confrontation simple, le participant est invité à commenter l'enregistrement de sa propre activité (Mollo, \& Falzon, 2004). En amont, des « traces de l'activité » du professionnel sont recueillies: des notes ethnographiques, des enregistrements audios, des enregistrements vidéo, des photographies, etc. Dans cette étude, les supports des entretiens sont plutôt des « représentations de l'activité ». L'autoévaluation de la vigilance et de la qualité des soins, ainsi que les choix des stratégies de régulations, sont commentées a posteriori par l'infirmier. Cette verbalisation est cruciale pour contextualiser ses réponses et repérer les facteurs qui les ont potentiellement influencées. L'entretien est donc divisé en 3 parties : la première sur l'auto-évaluation de la vigilance, la deuxième sur l'auto-évaluation de la qualité des soins et la troisième sur les stratégies de régulation. Chaque partie est introduite avec une phrase précise.

- Vigilance : Que penses-tu de ces réponses? 
- Qualité des soins : J'aimerais que tu reprennes chacun de ces critères et que tu m'expliques ton choix de couleur.

- Stratégies de régulation: J'aimerais que tu reprennes chacune de ces stratégies et que tu m'expliques pourquoi tu as choisi de les cocher ou de ne pas les cocher.

Ses réponses sous les yeux, le participant est invité à se remémorer le contexte dans lequel il a rempli les échelles et le questionnaire. Les données d'observation de l'activité notées en continu constituent un support supplémentaire pour préciser le discours de l'interviewé. Par exemple, l'infirmier explique qu'il a fait appel à ses collègues (stratégie $n$ - 8) en confiant la préparation d'un soin à un autre infirmier. L'intervenante a observé ce transfert de tâche et demande à l'infirmier s'il s'agissait bien d'une seringue d'héparine à $22 \mathrm{~h}$ 30. Les données d'observation permettent également de relancer le discours. Par exemple, l'intervenante a observé à $3 \mathrm{~h}$ du matin que l'infirmier s'est rendu dans un autre module alors que l'infirmier n'a pas coché la stratégie « prendre une pause à l'extérieur du module » (stratégie $n^{\circ} 11$ ). Elle lui demande alors d'expliquer pourquoi il s'y est rendu. Les témoignages recueillis ont été analysées sur Word. Soumis à plusieurs lectures, ils ont été classés dans différentes catégories. Pour chacune d'elles le nombre de participants concernés a été comptabilisé et divisé par le nombre total de participants, ce qui a permis d'obtenir des pourcentages.

\section{Résultats}

Les résultats de l'étude sont présentés de la même manière : ceux des questionnaires dans un premier temps et ceux des entretiens dans un second temps. Au vu du grand nombre de données produites par les entretiens, nous avons fait le choix de ne présenter que les catégories de témoignages exprimées par plus de $20 \%$ des infirmiers.

\subsection{Les infirmiers s'estiment de moins en moins vigilants au fil du poste de nuit de 12 heures}

41 L'indice de vigilance des infirmiers diminue significativement au cours des 12 heures du poste de nuit $\left(\chi^{2}=46,85 ; p=0\right)$ (Fig.3). 
Figure 3. Niveau de vigilance estimé par les infirmiers aux 4 temps de passation de l'échelle d'autoestimation « Activation-Deactivation Adjective Checklist " (Thayer, 1967) (22h, 01h, 04h et 06h). Figure 3. Level of alertness estimated by nurses the 4 times they completed the "Activation-Deactivation Adjective Checklist" (Thayer, 1967) (22:00, $01: 00,04: 00$ and $06: 00)$

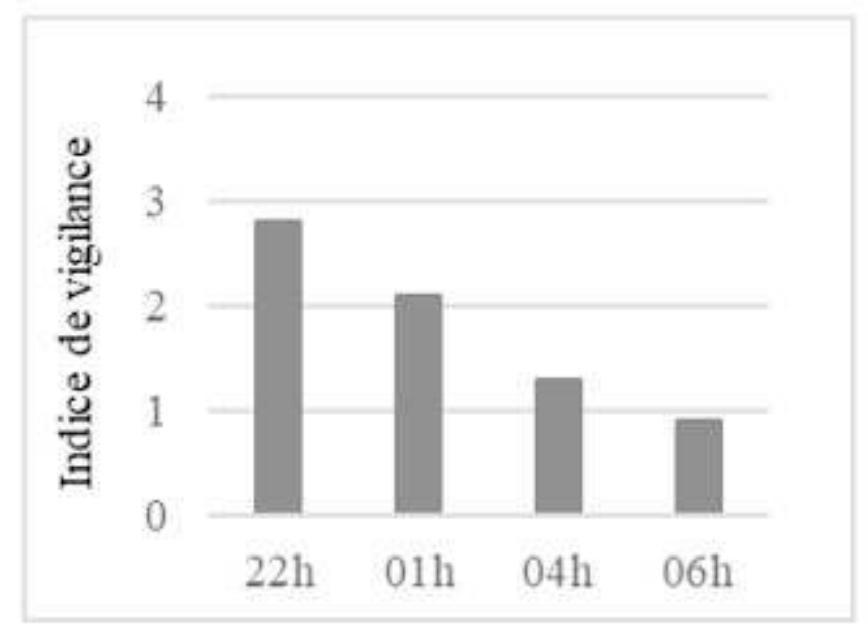

Le contenu des entretiens est présenté dans l'ordre suivant la chronologie du poste de travail (Tableau 3). Il montre qu'une partie des infirmiers considèrent que les niveaux d'énergie et de vigilance dépendent de la charge de travail. Entre $19 \mathrm{~h}$ et $22 \mathrm{~h}, 33 \%$ d'entre eux expliquent se sentir énergiques et vigilants grâce à la forte activité qui prédomine aux premières heures du poste. Comme le montre la présentation chronologique des tâches de l'infirmier sur un poste de nuit de 12 heures (Figure 1), la charge de travail diminue progressivement en deuxième partie de nuit (après le relevé des constantes à $00 \mathrm{~h}$ ). Cette diminution entraine une baisse d'énergie selon $42 \%$ des infirmiers. D'après les propos recueillis entre $1 \mathrm{~h}$ et $6 \mathrm{~h}, 50 \%$ des infirmiers luttent pour ne pas s'endormir et $33 \%$ se laissent gagner par la somnolence. $25 \%$ des infirmiers soulignent qu'ils restent attentifs aux alarmes malgré l'augmentation de leur somnolence.

Tableau 3. Commentaires des infirmiers sur les réponses données dans l'échelle d'auto-estimation " Activation-Desactivation Adjective Checklist » (Thayer, 1967) sur l'ensemble du poste de nuit en 12 heures (Partie 1).

Table 3. Nurses' comments concerning the answers given in the "Activation-Deactivation Adjective Checklist" (Thayer, 1967) over the 12-hour night shift (Part 1)

\begin{tabular}{|c|c|c|}
\hline & Catégorie de témoignage & $\begin{array}{c}\% \\
\text { infirmiers }\end{array}$ \\
\hline - & $\begin{array}{l}\text { Je me sens énergique et vigilant(e) parce qu'il y a une forte } \\
\text { activité qui prédomine sur les premières heures du poste (19h- } \\
22 \mathrm{~h} \text { ). }\end{array}$ & $33 \%$ \\
\hline & $\begin{array}{l}\text { Mon énergie diminue parce que j'ai de moins en moins de choses } \\
\text { à faire. }\end{array}$ & $42 \%$ \\
\hline & Je lutte pour ne pas m'endormir (01h-06h). & $50 \%$ \\
\hline , & Je me laisse gagner par la somnolence $(01 \mathrm{~h}-06 \mathrm{~h})$. & $33 \%$ \\
\hline כ & $\begin{array}{l}\text { Même si je suis somnolent, je reste attentif aux alarmes } \\
\text { environnantes. }\end{array}$ & $25 \%$ \\
\hline
\end{tabular}




\subsection{Les infirmiers qui travaillent ponctuellement de nuit se sentent moins vigilants que les infirmiers qui travaillent de nuit toute l'année}

Le niveau de vigilance est significativement différent entre les infirmiers fixes de nuit et les infirmiers qui travaillent de nuit ponctuellement $\left(\chi^{2}=5 ; p=0,025\right)$. L'indice de vigilance moyen est à 2,3 chez les infirmiers fixes et 1,5 chez les infirmiers ponctuels.

Les entretiens révèlent que parmi tous les infirmiers qui luttent pour ne pas s'endormir entre $1 \mathrm{~h}$ et $6 \mathrm{~h}, 67 \%$ d'entre eux travaillent ponctuellement de nuit. Tous les infirmiers qui ferment les yeux et se laissent gagner par la somnolence travaillent ponctuellement de nuit (Tableau 4).

Tableau 4. Commentaires des infirmiers sur les réponses données dans l'échelle d'auto-estimation "Activation-Desactivation Adjective Checklist » (Thayer, 1967) sur l'ensemble du poste de nuit en 12 heures (Partie 2).

Table 4. Nurses' comments concerning the answers given in the "Activation-Deactivation Adjective Checklist" (Thayer, 1967) over the 12-hour night shift (Part 2)

\begin{tabular}{|c|l|c|c|}
\hline \multirow{2}{*}{$\begin{array}{c}\text { Entre } \\
01 \mathrm{~h}\end{array}$} & \multicolumn{1}{|c|}{ Catégorie de témoignage } & \multicolumn{2}{|c|}{$\%$ infirmiers } \\
\cline { 3 - 4 } $\begin{array}{c}\text { et } \\
06 \mathrm{~h}\end{array}$ & Je lutte pour ne pas m'endormir. & $67 \%$ & $33 \%$ \\
\cline { 2 - 4 } & Je me laisse gagner par la somnolence. & $100 \%$ & $0 \%$ \\
\hline
\end{tabular}

\subsection{Les critères de qualité des soins sont évalués positivement par les infirmiers tout au long du poste}

L'auto-évaluation de la totalité des critères de qualité des soins réunis n'est pas différente entre les quatre moments du poste de nuit $\left(\chi^{2}=1,05 ; p=0,79\right)$ et se maintient à un score très positif (Figure 4). Pourtant l'auto-évaluation du critère de concentration (Q09) diminue significativement au cours du poste $\left(\chi^{2}=20,53 ; p=0\right)$ tout en restant à un niveau positif (Figure 5). 
Figure 4. Score d'auto-évaluation moyen des 11 critères de qualité réunis aux 4 temps de passation du questionnaire de qualité $(22 \mathrm{~h}, 01 \mathrm{~h}, 04 \mathrm{~h}$ et $06 \mathrm{~h})$.

Figure 4. Average self-assessment score for the 11 quality criteria collected the 4 times they completed the quality questionnaire (22:00, 01:00, 04:00 and 06:00)

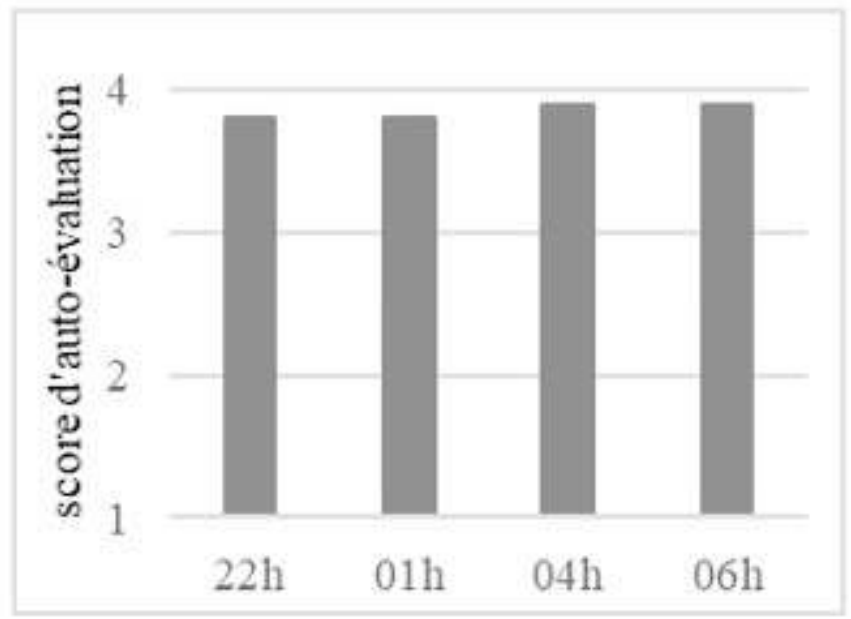

Figure 5. Score d'auto-évaluation moyen des critères de qualité Q09 «Etes-vous resté(e) concentré (e) ? ", Q10 « Etes-vous certain(e) de n'avoir rien oublié ? » et Q11 «Etes-vous certain(e) de ne pas vous êtes trompé(e) ? " aux 4 temps de passation du questionnaire de qualité $(22 \mathrm{~h}, 01 \mathrm{~h}, 04 \mathrm{~h}$ et $06 \mathrm{~h})$.

Figure 5. Average self-assessment score for the quality criteria : Q09 "Have you stayed focused ?", Q10 "Are you sure that you did not forget anything ?" and Q11 "Are you sure that you did not make any mistakes?" the 4 times they completed the quality questionnaire (22:00, 01:00, 04:00 and 06:00)

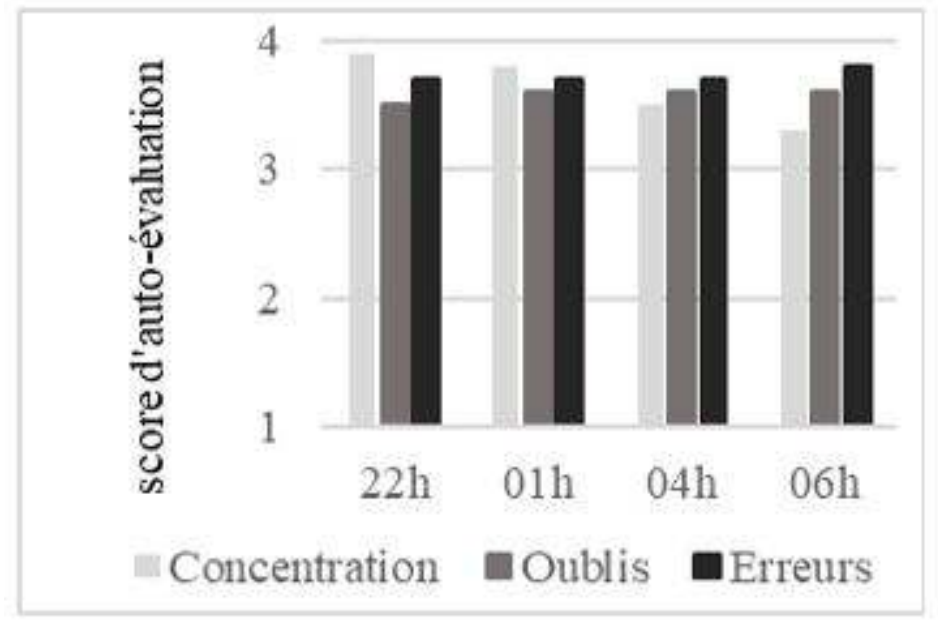

D'après les entretiens (Tableau 5), l'accomplissement des tâches (Q01) est un critère de qualité que tous les infirmiers associent en priorité aux tâches prévues et imprévues du début du poste de nuit. Chez $33 \%$ d'entre eux, le critère est atteint lorsqu'il n'y a plus qu'à surveiller les patients, répondre à leurs besoins et relever les constantes. Le respect des règles d'hygiène et de sécurité (Q02) est un critère plus difficile à atteindre dans les situations d'urgences selon $29 \%$ des infirmiers. Concernant l'adéquation et la fonctionnalité du matériel (Q03), $29 \%$ des infirmiers disent qu'il doit être à disposition dans le module. Le critère de la prise en charge globale (Q04) combine plusieurs objectifs : la prise de connaissance de l'état du patient aux niveaux clinique et paraclinique (67\%), 
l'assurance des soins prescrits et de confort (58\%) et la vérification de son environnement (33\%). Chez $37 \%$ des infirmiers, la prise en charge reste positive si les patients sont stables. Le lien entre les prescriptions et l'état des patients (Q06) est assuré par la connaissance antérieure de l'infirmier sur les patients $(58 \%)$, la stabilité des patients et des prescriptions ( $54 \%$ ), la communication avec les médecins (42\%), la qualité des transmissions ( $25 \%$ ), la vérification de l'adéquation entre la feuille de réanimation et les paramètres de la chambre (21\%) et l'expérience de l'infirmier (21\%). Le critère de l'organisation (Q07) est atteint si l'infirmier a accompli ses tâches à l'heure et sans prendre de retard (37\%), s'il a anticipé la charge de travail, les imprévus potentiels et la baisse de vigilance (37\%), s'il ne s'est pas précipité ou dispersé (33\%) et s'il a pu prendre un temps de repos (21\%). Les imprévus sont anticipés (Q08) en s'avançant au maximum dans son travail selon $50 \%$ des infirmiers. Entre $1 \mathrm{~h}$ et $6 \mathrm{~h}, 54 \%$ des infirmiers expliquent que leur baisse de concentration (Q09) est liée à la diminution de la charge de travail et l'augmentation de la somnolence. $37 \%$ des infirmiers admettent qu'ils ne peuvent être totalement certains de n'avoir rien oublié ou de ne pas s'être trompés. Pourtant, le graphique (Figure 5) montre que leur auto-évaluation des oublis (Q10) et des erreurs (Q11) reste positive. $83 \%$ des infirmiers sont certains de n'avoir rien oublié ou de ne pas s'être trompés parce qu'ils vérifient les prescriptions en permanence.

Tableau 5. Commentaires des infirmiers sur les réponses données dans l'échelle de qualité sur l'ensemble du poste de nuit en 12 heures.

Table 5. Nurses' comments concerning the answers given in the quality scale over the 12-hour night shift

\begin{tabular}{|c|c|c|}
\hline $\begin{array}{l}\text { Critère de } \\
\text { qualité }\end{array}$ & Catégorie de tém oignage & $\begin{array}{c}\% \\
\text { in firmiers }\end{array}$ \\
\hline \multirow{3}{*}{$\begin{array}{c}\text { Q01 } \\
\begin{array}{l}\text { Accomplissement } \\
\text { des taches }\end{array}\end{array}$} & $\begin{array}{l}\text { Jai accompli toutes mes tiches du début de nuit, celles qui correspondent au } \\
\text { "g gros tour " et qui consistent a cadrer le module, ainsi que les täches qui se } \\
\text { sont rajoutees (19h-01h). }\end{array}$ & $100 \%$ \\
\hline & $\begin{array}{l}\text { J'atteins ce critère car ( }(0 \mathrm{~h} \text {-06h) : } \\
\text { - jen'ai plus qu'à relever les constantes toutes les } 2 \text { heures et surveiller mes } \\
\text { patients }\end{array}$ & $33 \%$ \\
\hline & $\begin{array}{l}\text { A partir de } 00 h 00, \text { la prise en charge } s \text { ' inscrit dans la continuité de la nuit et } \\
\text { reavoi à :- surveiller les patients } \\
\text {-répondre aux besoins éventuels des patients }\end{array}$ & $\begin{array}{l}33 \% \\
33 \%\end{array}$ \\
\hline $\begin{array}{l}\text { Q02 Respect des } \\
\text { régles d hygiène } \\
\text { et de sécurité }\end{array}$ & $\begin{array}{l}\text { Les règles d'hygiène sont plus difficiles à respecter dans les situations } \\
\text { d'urgences, où il faut travailler dans la précipitation. }\end{array}$ & $29 \%$ \\
\hline $\begin{array}{l}\text { Q03 Matériel } \\
\text { adéquat et } \\
\text { fonctionnel }\end{array}$ & Le matériel doit être à disposition dans le module. & $29 \%$ \\
\hline $\begin{array}{l}\text { Q04 Prise en } \\
\text { charge « globale * }\end{array}$ & 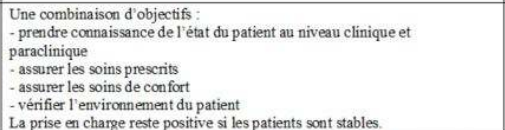 & $\begin{array}{l}67 \% \\
58 \% \\
58 \% \\
33 \% \\
37 \%\end{array}$ \\
\hline $\begin{array}{l}\text { Q06 Liens entre } \\
\text { prescriptions et } \\
\text { état des patients }\end{array}$ & 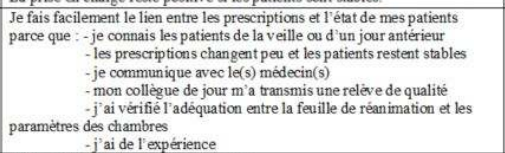 & $\begin{array}{l}58 \% \\
54 \% \\
42 \% \\
22 \% \% \\
21 \% \\
21 \%\end{array}$ \\
\hline Q07 Organisation & 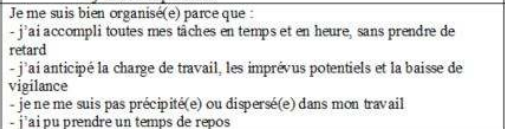 & $\begin{array}{l}71 \% \\
37 \% \\
33 \% \\
21 \%\end{array}$ \\
\hline $\begin{array}{l}\text { Q08 Anticipation } \\
\text { et gestion des } \\
\text { imprevus }\end{array}$ & J anticipe I 'imprévu en mavançant au maximum dans mon travail. & $50 \%$ \\
\hline $\begin{array}{c}\text { Q09 } \\
\text { Concentration }\end{array}$ & $\begin{array}{l}\text { Quand la charge de travail diminue et que ma somnolence augmente, j'ai } \\
\text { plus de mal à me concentrer (01h- }-06 \mathrm{~h} \text { ). }\end{array}$ & $54 \%$ \\
\hline \multirow{2}{*}{$\begin{array}{l}\text { Q10 Oublis } \\
\text { Q11 Erreurs }\end{array}$} & $\begin{array}{l}\text { On ne peut jamais être certain de ne rien avoir oublié ou de ne pas s'être } \\
\text { trompé }\end{array}$ & $37 \%$ \\
\hline & $\begin{array}{l}\text { Je suis certain(e) den navoir rien oublié ou de ne pas m'être trompé(e) car je } \\
\text { vérifie les prescriptions en pemanence }\end{array}$ & $83 \%$ \\
\hline
\end{tabular}




\subsection{Des stratégies pour " gérer le travail » en 1 ère partie de nuit et des stratégies pour « gérer l'état d'éveil » en 2 ème partie de nuit}

Douze stratégies de régulation ont été proposées aux infirmiers. Les 9 premières stratégies visent une gestion du travail tandis que les 3 dernières une gestion de l'état d'éveil.

\subsubsection{Stratégies pour gérer le travail}

Figure 6. Score moyen d'utilisation des stratégies S01 « Accélérer sa cadence de travail "; S02 "Ralentir sa cadence de travail »; S03 "Fonctionner par priorités »; S04 " S'avancer dans son travail »; S05 « Utiliser un support écrit personnel »; S06 "Vérifier plusieurs fois la feuille de réanimation »; S07 "S'organiser mentalement »; S08 " Faire appel aux collègues »; S09 "Repousser ou réduire son temps de pause " aux 4 temps de passation du questionnaire des stratégies de régulation ( $22 \mathrm{~h}, 01 \mathrm{~h}, 04 \mathrm{~h}$ et $06 \mathrm{~h})$.

Figure 6. Average score for utilization of regulation strategies:S01 "Increase work rate"; S02 "Reduce work rate"; SO3 "Prioritize"; SO4 "Move ahead with work"; SO5 "Use a personal written medium"; S06 "Check the reanimation sheet several times"; S07 "Mental organization"; S08 "Ask colleagues for help"; S09 "Postpone break or reduce its duration" the 4 times they completed the regulation strategy questionnaire $(22: 00,01: 00,04: 00$ and 06:00)

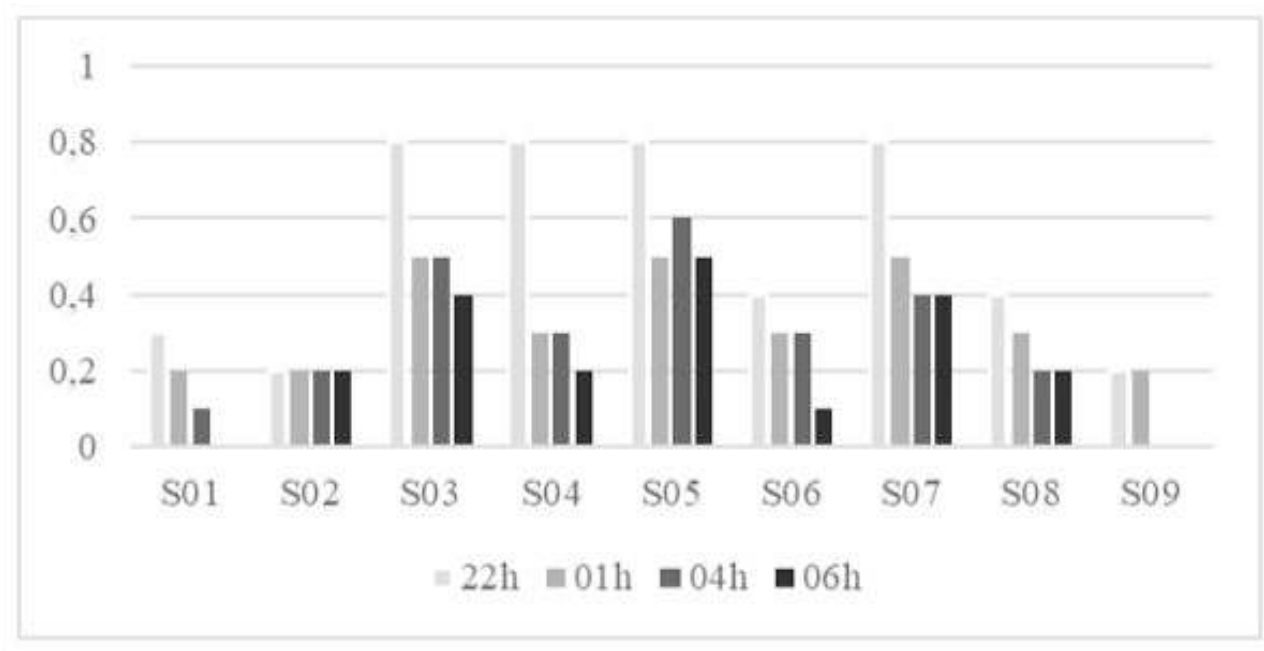

Le score d'utilisation n'est pas significativement différent entre les 4 moments du poste pour les stratégies «Ralentir sa cadence de travail» (S02), «Faire appel aux collègues" (S08) et "Trouver des occupations" (S10). En revanche, les stratégies suivantes présentent un score d'utilisation significativement différent :

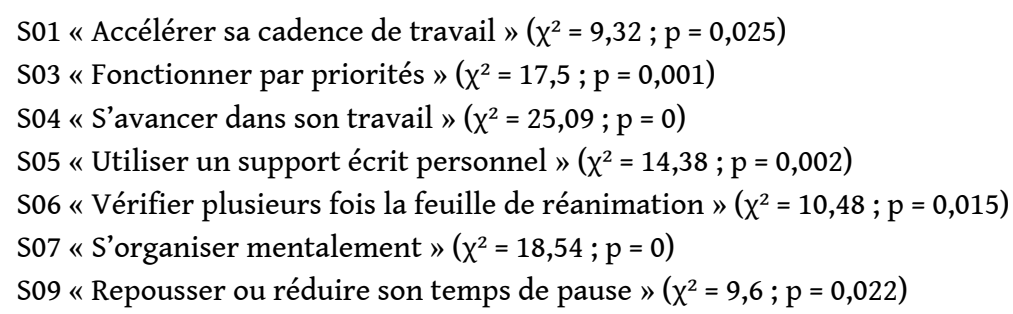

D'après le graphique (Figure 6), c'est entre $19 \mathrm{~h}$ et $22 \mathrm{~h}$ que ces stratégies sont les plus utilisées par rapport au reste du poste. La stratégie « Repousser ou réduire son temps de pause » (S09) est utilisée au même niveau jusqu'à 01h. Plus les heures passent, moins ces stratégies sont utilisées. 


\subsubsection{Stratégies pour gérer l'état d'éveil}

Figure 7. Score d'utilisation moyen des stratégies S10 «Trouver des occupations "; S1 1 " Prendre une pause à l'extérieur du module » et $\mathrm{S} 12$ « Se reposer dans le module » aux 4 temps de passation du questionnaire des stratégies de régulation $(22 \mathrm{~h}, 01 \mathrm{~h}, 04 \mathrm{~h}$ et $06 \mathrm{~h})$.

Figure 7. Average score for utilization of regulation strategies: S10 "Keep busy"; $S 11$ "Take a break outside the care module" and S12 "Have a rest in the care module" the 4 times they completed the regulation strategy questionnaire (22:00, 01:00, 04:00 and 06:00)

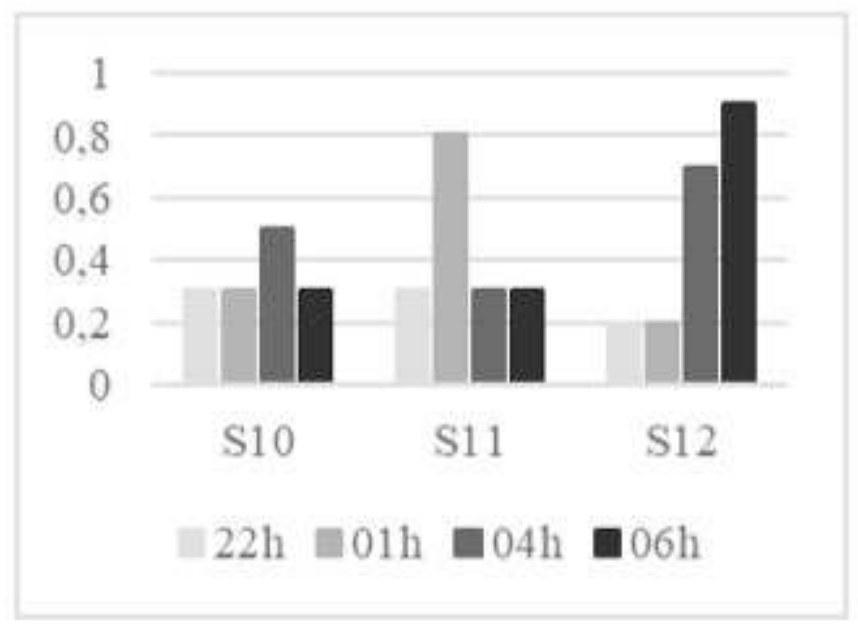
différent entre les 4 moments du poste :

S11 « Prendre une pause à l'extérieur du module» $\left(x^{2}=20,8 ; p=0\right)$

S12 « Se reposer dans le module $»\left(\chi^{2}=33,4 ; p=0\right)$

D’après le graphique (Figure 7), la stratégie « Se reposer dans le module » (S12) est de plus en plus utilisée, notamment dans la deuxième partie du poste de nuit. La stratégie «Prendre une pause à l'extérieur du module » (S11) correspond au temps de repas pris par tous les infirmiers entre $22 \mathrm{~h}$ et $1 \mathrm{~h}$.

\subsubsection{Témoignages recueillis en entretien}

Les stratégies S01 «Accélérer sa cadence de travail », S03 « Fonctionner par priorités ", S04 "S'avancer dans son travail», S05 «Utiliser un support écrit personnel» et S07 «S'organiser mentalement» contribuent à l'atteinte des objectifs liés au critère d'organisation (Q07). L'accélération de la cadence de travail (S01) vise à anticiper les imprévus et les pics d'activité selon $25 \%$ des infirmiers. D’après les témoignages liés au questionnaire des stratégies de régulation (Tableau 6), la stratégie "S'avancer dans son travail " (S04) renvoie à toute une série de tâches effectuées aux premières heures du poste. En début de nuit, les infirmiers s'avancent en sortant et/ou préparant tous les traitements et les soins (bilans sanguins, seringues, perfusions, etc.) pour $83 \%$ et en préparant les bilans sanguins et les feuilles de réanimation du lendemain pour $50 \%$. Les infirmiers disent que s'avancer revient à anticiper: $54 \%$ d'entre eux s'avancent pour anticiper un imprévu et/ou une surcharge de travail ; $54 \%$ pour sécuriser la prise en charge du patient et $25 \%$ pour anticiper la baisse de vigilance. Entre $1 \mathrm{~h}$ et $6 \mathrm{~h}, 54 \%$ des infirmiers expliquent n'avoir plus besoin de s'avancer puisqu'ils l'ont fait en début de nuit et qu'il ne reste plus qu'à surveiller les patients et administrer les soins prévus au 
moment venu. Dans la même perspective, 50 \% des infirmiers ne fonctionnent plus par priorités si la charge de travail se limite à la surveillance des patients et qu'il n'y a plus d'imprévus à gérer. Concernant le support écrit personnel (S05), qui est utilisé par $58 \%$ des infirmiers, $33 \%$ en font un récapitulatif du dossier des patients, $33 \%$ une planification écrite et personnelle des soins pour visualiser leur charge de travail et réduire leurs déplacements entre les feuilles de réanimation et les paillasses. Au début du poste de nuit, $37 \%$ de infirmiers disent vérifier plusieurs fois la feuille de réanimation (S06) pour relever les rajouts potentiels de prescriptions, $29 \%$ pour contrôler les prescriptions au moment de préparer et d'administrer les soins et $25 \%$ pour valider les soins effectués. À propos de l'organisation mentale (S07), $62 \%$ des infirmiers disent qu'ils savent ce qu'ils ont à faire et qu'ils font régulièrement le point sur ce qu'ils ont fait, sur ce qu'ils ont à faire et sur la façon dont ils vont procéder. À partir de 1 h, $21 \%$ des infirmiers disent n'avoir plus besoin de s'organiser mentalement parce que la plupart des tâches ont été faites en début de nuit. Pour ce qui est de l'entraide entre collègues (S08), $21 \%$ des infirmiers disent confier une ou plusieurs tâche(s) à un ou plusieurs collègue(s) pour continuer leur travail et éviter de prendre du retard.

Tableau 6. Commentaires des infirmiers sur les réponses données dans le questionnaire des stratégies de régulation (de S01 à S09) sur l'ensemble du poste de nuit en 12 heures.

Table 6. Nurses' comments concerning the answers given in the regulation strategy questionnaire (from S01 to S09) over the 12-hour night shift

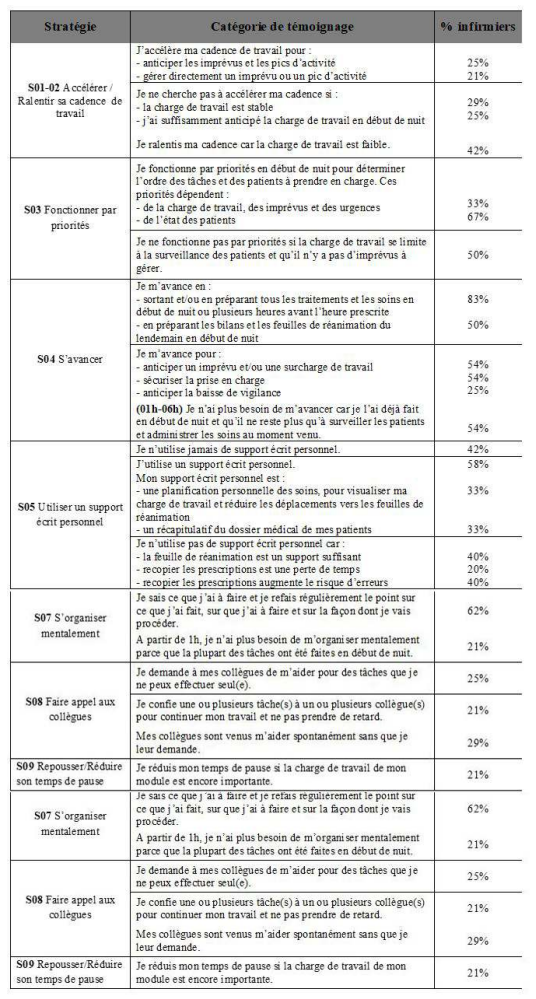

53 D'après les commentaires liés au questionnaire des stratégies de régulation (Tableau 7), $58 \%$ des infirmiers s'occupent (S10) en se consacrant à une activité personnelle et $25 \%$ en étalant leurs tâches. En début de nuit, les infirmiers ne cherchent pas à s'occuper parce qu'ils se sentent en forme pour $60 \%$ et parce qu'ils sont suffisamment occupés par la charge de travail pour $53 \%$. C'est entre $1 \mathrm{~h}$ et $6 \mathrm{~h}$ que la stratégie «Se reposer dans le module» (S12) est la plus utilisée (Figure 7); $62 \%$ des infirmiers considèrent qu'ils se 
reposent en s'asseyant et/ou en allongeant les jambes, $52 \%$ en somnolant. $71 \%$ des infirmiers disent que la prise de repos permet de soulager le corps et récupérer de l'énergie et de la concentration pour le travail restant.

Tableau 7. Commentaires des infirmiers sur les réponses données dans le questionnaire des stratégies de régulation (de S10 à S12) sur l'ensemble du poste de nuit en 12 heures.

Table 7. Nurses' comments concerning the answers given in the regulation strategy questionnaire (from S10 to S12) over the 12-hour night shift

\begin{tabular}{|c|c|c|}
\hline Stratégie & Catégorie de témoignage & $\%$ infirmiers \\
\hline \multirow{2}{*}{$\begin{array}{l}\text { S10 Trouver des } \\
\text { occupations }\end{array}$} & $\begin{array}{l}\text { Quand je cherche à m'occuper: } \\
\text { - je me consacre à une activité personnelle (téléphone, lecture, } \\
\text { discussion entre collègues) } \\
\text { - j'étale mes tâches pour avoir toujours quelque chose à faire }\end{array}$ & $58 \%$ \\
\hline & $\begin{array}{l}\text { Pour ne pas m'endormir. } \\
\text { En début de nuit, je ne cherche pas à m'occuper car: } \\
\text { - je me sens en forme } \\
\text { - je suis suffisamment occupé par la charge de travail }\end{array}$ & $\begin{array}{l}29 \% \\
60 \% \\
53 \%\end{array}$ \\
\hline \multirow{2}{*}{$\begin{array}{l}\text { S11 Prendre une pause à } \\
\text { l'extérieur du module }\end{array}$} & Pause diner & $71 \%$ \\
\hline & $\begin{array}{l}\text { Café, cigarette, discussion entre collègues, } s^{\prime} \text { informer de la } \\
\text { charge de travail des autres, prendre l'air. }\end{array}$ & $46 \%$ \\
\hline \multirow{2}{*}{$\begin{array}{l}\text { S12 Se reposer dans le } \\
\text { module }\end{array}$} & $\begin{array}{l}\text { Je me repose quand: } \\
\text { - je m'assois, j'allonge les jambes } \\
\text { - je somnole }\end{array}$ & $\begin{array}{l}62 \% \\
52 \%\end{array}$ \\
\hline & $\begin{array}{l}\text { En me reposant, je soulage mon corps, je récupère de l'énergie } \\
\text { et je me sens plus concentrée pour le travail qu'il reste à faire. }\end{array}$ & $71 \%$ \\
\hline
\end{tabular}

\section{Discussion}

\subsection{La diminution de la vigilance confirmée}

54 La diminution significative de la vigilance perçue par les infirmiers au cours du poste de nuit de 12 heures n'est pas surprenante au regard des nombreuses études ayant montré que le travail posté incluant la nuit était associé à une baisse des performances cognitives (Anses, 2016). Nous expliquons cette diminution par le double effet de la désynchronisation circadienne (travailler de nuit) et de la durée du poste (travailler 12 heures d'affilée) (Folkard, \& Åkerstedt, 1992). Elle est également liée à la rythmicité temporelle des tâches. En effet, la majorité des soins techniques et de confort sont administrés en première partie de nuit, entre $20 \mathrm{~h}$ et $00 \mathrm{~h}$ (Figure 1). S'il n'y a pas d'urgence(s) à gérer, la charge de travail diminue progressivement. Cette tendance se conjugue à la désynchronisation circadienne du travail de nuit : l'infirmier "se relâche " et se met à lutter contre le déclin de ses propres capacités psychophysiologiques.

\subsection{Un « scénario de travail » construit en début de nuit sur des stratégies d'anticipation}

D'après nos résultats, les 7 stratégies suivantes présentent un score d'utilisation significativement différent entre les 4 moments du poste de nuit : accélérer sa cadence de travail, prioriser, s'avancer, utiliser un support écrit personnel, vérifier plusieurs fois la feuille de réanimation, s'organiser mentalement et repousser ou réduire son temps de pause (cf. paragraphe 9.5). Elles sont plus utilisées entre $19 \mathrm{~h}$ et $22 \mathrm{~h}$ que par rapport au 
reste du poste, exceptée la stratégie de repousser ou réduire son temps de pause dont l'utilisation se prolonge jusqu'à $1 \mathrm{~h}$. Ces stratégies sont de moins en moins utilisées au fil des heures (Figure 6). Elles incarnent l'organisation anticipée du travail des infirmiers qui vise à "cadrer le service", autrement dit maîtriser le processus. Les entretiens (Tableau 6) montrent en effet que les infirmiers cherchent à accomplir toutes les tâches prévues et imprévues en début de nuit, pour n'avoir plus qu'à surveiller les patients, répondre à leurs besoins et relever les constantes sur le reste de la nuit. Ils considèrent qu'ils se sont bien organisés s'ils ont accompli toutes leurs tâches en temps et en heure sans prendre de retard, s'ils ne se sont pas précipités ou dispersés, et s'ils ont anticipé la charge de travail et les imprévus potentiels. Le travail hospitalier s'inscrivant dans un environnement dynamique, il est en permanence soumis à l'imprévisibilité. Un infirmier sur deux déclare qu'il anticipe l'imprévu en s'avançant un maximum dans son travail (Tableau 6). Ainsi, l'accélération de la cadence, la priorisation et l'avancement des tâches visent simultanément l'anticipation des imprévus et de la surcharge de travail. Les infirmiers cherchent à optimiser le temps dont ils disposent. Il ne s'agit ni d'être en retard, ni d'être dans les temps, mais plutôt d'être en avance. Plus l'infirmier a de l'avance, moins il a de tâches en suspens et plus il a une vision précise de la situation de travail et de ses marges de manœuvre. Nous sommes bien dans le contrôle de processus expliqué par Van Daele et Carpinelli (2001) qui citent les mêmes objectifs : être dans le temps, ne pas se faire déborder, avoir une marge de manœuvre et être en avance plutôt qu'en retard.

Les entretiens (Tableau 6) montrent qu'au début du poste de nuit, les infirmiers vérifient plusieurs fois la feuille de réanimation pour relever les rajouts potentiels de prescriptions, contrôler les prescriptions au moment de préparer et d'administrer les soins et valider les soins effectués. Ces vérifications répétées visent à établir un état des lieux de la charge de travail dès la pise de poste. Toupin (2008) a déjà constaté en unité de pneumologie que les infirmières s'assurent dès le début du poste de nuit que les prescriptions médicales permettent de prendre en charge les éventuelles crises d'angoisse des patients. Suite à cet état des lieux, les entretiens révèlent que les infirmiers sortent sur leurs paillasses tous les traitements et les soins prévus pour la nuit (bilans sanguins, seringues, perfusions, etc.), les préparent pour certains, et préparent également les tâches du lendemain matin (bilans sanguins et nouvelles feuilles de réanimation). Le fait de sortir tous les soins et les traitements prévus pour la nuit sur la paillasse permet déjà de se construire une représentation de la charge de travail à venir. Cette représentation s'organise et se renouvelle mentalement. D'après les entretiens, les infirmiers savent ce qu'ils ont à faire et refont régulièrement le point sur ce qu'ils ont fait, sur ce qu'ils ont à faire et sur la façon dont ils vont procéder: ils réactualisent mentalement leur représentation de la situation de travail. Par ailleurs, cette représentation peut être matérialisée par un support écrit personnel, pour résumer les informations liées aux patients et/ou planifier et visualiser les tâches à réaliser.

Nous identifions à travers toutes ces stratégies de régulation la recherche d'une visibilité élargie sur la charge de travail à venir. Ces stratégies d'anticipation permettent aux infirmiers de se construire un scénario de travail, qui s'apparente à une planification de l'activité en produisant une représentation qui précède l'exécution (Van Daele, \& Carpinelli, 2001). Ce scénario peut d'ailleurs être réadapté selon les aléas du travail réel, lorsque l'infirmier est déjà dans l'exécution de la tâche et qu'il doit faire face à un imprévu. Pour reprendre les concepts de Falzon (2004), nous considérons que la 
construction du scénario de travail au début du poste de nuit repose sur une évaluation anticipée du système et s'inscrit donc dans la régulation "en boucle courte »; lorsque le scénario est réadapté selon les aléas du travail réel, il s'inscrit dans la régulation «en boucle longue ».

\subsection{Un scénario de travail déroulé sur le reste du poste pour gérer la baisse de vigilance}

D'après nos résultats les infirmiers s'avancent non seulement pour anticiper les imprévus et la surcharge de travail, mais aussi pour anticiper la baisse de vigilance et sécuriser la prise en charge (Tableau 6). L'anticipation de la vigilance fait d'ailleurs partie d'une bonne organisation selon les infirmiers (Tableau 5). En avançant la préparation des soins et des traitements aux moments où ils sont disponibles et vigilants, c'est-à-dire en début de nuit, les infirmiers éliminent le risque de les préparer précipitamment pendant un pic d'activité ou lorsque leur vigilance est faible. Cette préparation anticipée fait écho à l'étude de Prunier-Poulmaire (2008) dans laquelle les agents de douanes effectuent toutes les tâches exigeant le plus d'attention en début de nuit.

Nos résultats montrent également que l'entraide entre collègues est une stratégie dont l'utilisation ne diffère pas entre les moments du poste, ce qui signifie qu'elle peut être utilisée tout au long de la nuit (cf. paragraphe 9.5). En entretien, plusieurs infirmiers expliquent qu'ils confient une ou plusieurs tâche(s) à leurs collègues pour continuer leur travail et éviter de prendre du retard (Tableau 6). Le scénario peut donc favoriser le travail collectif : la préparation anticipée d'une tâche permet à l'infirmier de la confier à un collègue en cas de surcharge de travail. En service de néonatologie, Barthe (1999) a déjà identifié ce transfert de tâches (redistribution des enfants) entre les soignantes pour équilibrer mutuellement leur charge de travail. Dans ce service de réanimation, le transfert d'une tâche d'un infirmier à un autre se fait en toute confiance puisque qu'elle a été préparée en début de nuit par un opérateur disponible et concentré. Le scénario de travail peut alors éviter aux infirmiers de se concentrer sur plusieurs tâches à la fois ou de travailler dans la précipitation, et réduire potentiellement le risque d'erreurs et d'accidents.

D'après les résultats, les infirmiers considèrent qu'ils se sont bien organisés s'ils ont anticipé la baisse de vigilance et s'ils ont pu prendre un temps de repos (Tableau 5). C'est entre $1 \mathrm{~h}$ et $6 \mathrm{~h}$ que la stratégie de la prise de repos est la plus utilisée (Figure 7). Dans les entretiens (Tableau 7) les infirmiers considèrent qu'ils se reposent lorsqu'ils s'assoient et/ ou allongent les jambes, ou lorsqu'ils somnolent. Ce temps de repos leur permet de soulager leur corps, récupérer de l'énergie et se sentir plus concentré pour le travail restant. En prenant compte de l'augmentation importante des accidents de travail aux dernières heures du poste en 12 heures (Folkard, \& Tucker, 2003 ; Marias, \& al., 1996) et $\mathrm{du}$ risque de s'endormir au volant sur le trajet du retour au domicile (Heslegrave, \& al., 2000, in Knauth, 2007), ce temps de repos est un aménagement pouvant soutenir la sécurité des infirmiers. Le scénario de travail rend compatible l'accomplissement des tâches et cette prise de repos dans la deuxième partie de la nuit. Les entretiens montrent que la plupart des infirmiers sont convaincus des effets bénéfiques du temps de repos sur la vigilance (Tableau 7). Ils s'autorisent à le prendre parce qu'ils déroulent comme prévu leur scénario de travail. À condition que la charge de travail soit stable, la prise de repos des infirmiers peut s'intégrer à l'organisation du poste puisque la plupart des tâches 
exigeantes mentalement ont été avancées en début de nuit et donc fiabilisées avant le temps de repos.

\subsection{Un scénario de travail qui peut se trouver en désaccord avec certaines règles hospitalières}

61 Le scénario de travail des infirmiers est basé sur la préparation anticipée des soins et des traitements. Mais pour des raisons «médico-économiques ", plusieurs tâches devraient être exécutées plus tard dans la nuit. Selon la règle hospitalière, un bilan sanguin doit être préparé et étiqueté juste avant le prélèvement. Le pré-étiquetage du bon et des tubes n'est pas admis car il peut conduire à des erreurs (identité du patient, concordance entre les prescriptions et les tubes). La règle est la même pour une seringue. Les produits contenus dans les seringues comme l'héparine, l'insuline et certains antibiotiques ont une durée d'efficacité limitée une fois qu'ils sont préparés. La préparation anticipée des seringues n'est donc également pas approuvée. Par ailleurs, les médecins sont susceptibles de changer les prescriptions. Préparer à l'avance revient à prendre le risque de gaspiller le traitement ou de commettre une erreur de suivi.

62

Le temps de repos que s'aménagent les soignants en deuxième partie de nuit est aussi un sujet de polémique. En France, la législation sur le temps de repos au travail est encore limitée : les agents en horaires continus et qui ne doivent pas quitter leur poste de travail bénéficient d'un temps de pause de 20 minutes au maximum si le temps de travail est supérieur à 6 heures consécutives. Rien n'est précisé sur le fait de dormir au travail. Ces règles prescrites sont en contradiction avec le scénario de travail, qui repose sur une préparation anticipée des tâches pour faciliter une prise de repos durable sur l'espace de travail.

\subsection{Une perception positive de la qualité des soins tout au long du poste}

63 La qualité des soins est positivement évaluée par les infirmiers tout au long du poste. Ils se disent relativement certains de n'avoir rien oublié ou de ne pas s'être trompés. Et bien que l'auto-évaluation de leur concentration diminue, elle se maintient à un score positif. Ces résultats sont à rapprocher de l'étude de Tirilly, Barthe et Gentil (2015) menée dans un service de soins intensifs de cardiologie, et qui montre également que l'autoévaluation de la qualité des soins se maintient à un niveau très positif tout au long des postes de nuit. Ces résultats ne vont pas dans le sens des travaux ayant exploré le lien entre les postes longs et la diminution de la vigilance. Nous ne pouvons que nous interroger sur la valeur de l'auto-évaluation du travail accompli, qui repose sur les représentations subjectives des infirmiers, et non pas sur une analyse objective de la réalité. La difficulté à avoir une auto-évaluation précise des compétences professionnelles a été constatée dans plusieurs études menées en milieu médical. Hodges, Regehr et Martin (2001) ont étudié la capacité des résidents en médecine à autoévaluer leurs compétences relationnelles dans le cas de mauvaises nouvelles à annoncer à un patient : les résultats montrent une surestimation de leurs compétences dans ce domaine. Haun et al. (2000) ont trouvé des résultats similaires en demandant à des techniciens de laboratoires médicaux d'évaluer leurs connaissances sur les terminologies médicales et sur leur capacité à résoudre des problèmes de la vie courante au sein du laboratoire. 
Enfin, Weibel et al. (2014) signalent que les études réalisées sur les erreurs auto-déclarées paraissent minimiser l'effet de l'éveil prolongé sur la survenue d'erreurs. Pour étudier la qualité des soins et plus généralement le travail du soignant, Tirilly, Barthe et Gentil (2015) suggèrent de compléter la méthodologie avec des indicateurs objectifs. Elles proposent par exemple le temps que passent les soignants à vérifier une action accomplie ou le nombre de fois où le soignant doit se reprendre pour réaliser correctement une tâche. Elles suggèrent aussi de prendre en compte le point de vue du patient sur son confort et la qualité des soins qui lui sont donnés. Dans de futures perspectives de recherche, nous nous attacherons donc à analyser les résultats du travail hospitalier au moyen d'indicateurs variés, mêlant potentiellement des dimensions objectives et subjectives.

\subsection{Réflexion critique sur la méthodologie}

La méthodologie est critiquable sur certains points. Que ce soit en répondant aux questionnaires ou en commentant ses réponses en entretien, le participant se retrouve dans un cadre dont les frontières sont difficilement franchissables. En effet, certaines stratégies sont développées inconsciemment par l'opérateur et ne sont donc même plus considérées comme telles. Sur ce point, l'observation de l'activité et l'entretien sont particulièrement importants, et mériteraient d'être perfectionnés. Nous avons fait le choix d'employer l'observation globale de l'activité, sans utiliser de grille, et en nous déplaçant d'un module à l'autre. Inévitablement, cela suppose de "rater » certains éléments de l'activité car lorsque l'intervenante est dans un module ou une chambre, elle ignore ce qui se passe dans le module voisin ou la chambre voisine. Enfin, nous devons rappeler que l'intervenante recueille les données pendant 12 heures et qu'elle-même est impactée par la désynchronisation circadienne du travail de nuit. La baisse de concentration de l'intervenante a donc inévitablement impacté le recueil de données.

\subsection{Perspectives pragmatiques : établir un compromis pour instaurer un certain degré de souplesse temporelle et organisationnelle}

65 Les horaires $2 \times 12$ gagnent du terrain en milieu hospitalier, mais présentent des risques potentiels pour la qualité et la sécurité des soins. Dans ce contexte, le rôle joué par les soignants est crucial. Leurs régulations de l'activité de travail peuvent être vues d'un mauvais œil par la hiérarchie, celle-ci souhaitant avant tout se protéger d'un événement indésirable, qui peut aller de la simple erreur d'étiquetage ou de dosage au décès d'un patient. À cela s'ajoute l'accumulation des contraintes budgétaires (Baret, 2002) qui n'encourage pas non plus la préparation anticipée des soins et des traitements (risque de gaspillage). Malgré les contestations dont elles font l'objet, les régulations de l'activité effectuées par les soignants, constatées ici et dans bien d'autres études sur les horaires postés et de nuit (Barthe, 2015), nous semblent indispensables au maintien de la qualité des soins. Elles sont envisageables dans les situations de travail où les opérateurs disposent d'une certaine souplesse temporelle et organisationnelle. Toupin, Barthe et Prunier-Poulmaire (2013, p. 12) préconisent elles-mêmes l'introduction d'une plus grande souplesse dans la tâche prescrite, pour « laisser aux salariés des marges de manœuvre et une autonomie suffisante et acceptable du point de vue de la santé et de la sécurité, pour 
qu'ils puissent organiser leur activité ». Elles considèrent que cette autonomie constitue « un véritable enjeu de conception au niveau des horaires de travail» et qu'il ne s'agit «non pas de la tolérer, mais de la favoriser et de la construire».

Dans ce service de réanimation, nous recommandons une certaine autonomie temporelle et organisationnelle dans l'organisation du travail de nuit en 12 heures. Cette autonomie est déjà favorisée par le cadre infirmier, qui prône un «management par la confiance » en laissant à ses équipes la liberté de réorganiser leur activité de travail. Nous ne pouvons dénier que certaines stratégies de régulation employées par les soignants sont en contradiction avec les règles de l'établissement. Cette contradiction doit être discutée et gérée par les acteurs au niveau local. La question des horaires doit donc se régler au cas par cas, dans le cadre d'une intervention. Selon Quéinnec, Teiger et de Terssac (2008), il n'y a pas de mode d'organisation unique mais des «solutions adaptées aux situations concrètes » (p. 203). L'élaboration de solutions d'aménagement en matière d'organisation temporelle du travail passe par le compromis. Si ce dernier est indispensable, c'est parce que les actions d'amélioration ont des effets différenciés sur les acteurs de l'entreprise. Leur mise en œuvre est donc indissociable d'une concertation et d'une discussion entre ces différents acteurs. Dans le cadre de notre étude, nous sommes dans une négociation entre les contraintes de budget et de sécurité portées par l'établissement et les contraintes organisationnelles portées par le personnel soignant.

Par ailleurs, la recherche d'aménagement des horaires va de pair avec la prise en compte des caractéristiques de la situation de travail. Selon Barthe et Verdier (2004), un questionnement sur la nature des tâches à accomplir et sur leurs conditions d'exécution peut contribuer à " diminuer le poids des contraintes inhérentes aux horaires atypiques » (p. 57). Rappelons que Barthe (2009) insiste sur l'importante de prendre en compte les caractéristiques du travail à accomplir (physique ou plutôt cognitif, actif ou de surveillance, isolé ou en équipe, etc.). Il est également nécessaire de prendre en compte les caractéristiques de l'entreprise et des opérateurs en tant que «déterminants de l'activité de travail» (Barthe \& Verdier, 2004 ; p. 57). Du côté des travailleurs, plusieurs facteurs comme l'âge, les charges familiales, ou le temps d'exposition aux horaires postés vont impacter les effets du travail en 12 heures (Barthe, 2009).

Nos résultats montrent enfin que les infirmiers ponctuellement de nuit s'estiment moins vigilants que les infirmiers fixes de nuit (cf. paragraphe 9.3) et qu'ils se laissent plus facilement gagner par la somnolence (Tableau 4). Nous considérons que l'infirmier fixe de nuit tolère mieux la désynchronisation prolongée du poste de nuit en 12 heures, de par son expérience du poste et de son appartenance à un collectif solide. Concernant l'expérience, Toupin (2008) montre en service de pneumologie que l'infirmière de nuit développe des connaissances sur l'évolution de sa fatigue et de sa vigilance au cours du poste, et adapte - sur la base de ces connaissances - sa pratique pour se préserver physiquement tout en répondant à ses objectifs de travail. À propos du collectif, Barthe (1999) montre en service de néonatologie les régulations collectives de l'activité des soignantes: elles s'entraident (transfert d'enfants, accomplissement de tâches à plusieurs) pour diminuer mutuellement leur charge de travail et s'apporter des aides techniques ou des compétences spécifiques à certains moments du poste ; elles organisent leur activité collectivement pour s'aménager individuellement des temps de repos. Ainsi, l'expérience et le collectif de travail sont deux conditions qui permettent aux soignants en poste de nuit de 12 heures de développer une organisation adaptée aux variations de leur état psychophysiologique et aux exigences de travail. Pour favoriser la souplesse 
temporelle et organisationnelle dans le travail des soignants, nous préconisons donc un renforcement des équipes de nuit, ou plus particulièrement du collectif de travail. Ce renforcement peut être une solution supplémentaire pour compenser les effets des horaires atypiques sur la fiabilité et la santé (Barthe, 2015).

\section{BIBLIOGRAPHIE}

Andorre, V., \& Quéinnec, Y. (1996). La prise de poste en salle de contrôle de processus continu : approche chrono psychologique. Le Travail Humain, 59(4), 335-354.

ANSES. (2016). Evaluation des risques sanitaires liés au travail de nuit. Avis de l'Anses (Agence nationale de sécurité sanitaire de l'alimentation, de l'environnement et du travail). Rapport d'expertise collective. Juin 2016, Edition scientifique, 430 p. https://www.anses.fr/fr/system/ files/AP2011SA0088Ra.pdf

ATIH (2014). Analyse des Bilans sociaux des établissements publics de santé à fin 2012. http:// www.atih.sante.fr/sites/default/files/public/content/2445/note_bilan_social_2012.pdf

Baret, C. (2002). Hôpital : le temps de travail sous tension. Une comparaison Belgique, Italie, France, Grande-Bretagne, Pays-Bas et Suède. Sciences Sociales et Santé, 20(3), 75-107.

Barthe B., \& Quéinnec Y. (2005). Work activity during night shifts in a hospital's neonatal department: How nurses reorganize health care to adapt to their alertness decrease. Ergonomia, 27(2), 119-129.

Barthe, B. (1999). Gestion collective de l'activité de travail et variation de la vigilance nocturne : le cas d'équipes d'hospitalières de travail en postes de nuit longs. (Thèse de doctorat). Université Toulouse 2. Barthe, B. (2009). Les $2 \times 12$ heures : une solution au conflit de temporalités du travail posté ? Temporalités, 10.

Barthe, B., Boccara, V., Delgoulet, C., Gaillard, I., Meylan, S., \& Zara-Meylan, V. (2017). Outiller l'observation de l'activité de travail : quels processus de conception d'un dispositif technique? Actes du 52ème congrès de la SELF, 543-547. (Toulouse, 20-22 septembre).

Barthe, B., Quéinnec, Y., \& Verdier, F. (2004). L'analyse de l'activité de travail en postes de nuit : Bilan de 25 ans de recherches et perspectives. Le Travail Humain, 67(1), 41-61.

Barthe, B., Tirilly, G. Gentil, C., \& Toupin, C. (2016). Job demands and resting and napping opportunities and job demands for nurses during night shifts: impact on sleepiness and selfevaluated quality of healthcare. Industrial Heath, 54(2), 157-162

Barthe, B. (2015). La déstabilisation des horaires de travail, In Thébaud-Mony, A. Davezies, P. Vogel, L. \& Volkoff, S. (Eds.) Les risques du travail. 223-232. Paris : Éditions la Découverte.

Bonnefond, A. (2002). Approche expérimentale des avantages et inconvénients liés à l'introduction d'un sommeil de courte durée lors du poste de nuit chez le travailleur posté. (Thèse de doctorat). Université Louis Pasteur, Strasbourg.

Bris, V. (Le)., Tirilly, G., \& Toupin, C. (2015). Le rôle de l'expérience et du collectif en situation de relève de poste. Perspectives interdisciplinaires sur le travail et la santé, (17-2). 
Brun, B., Devaux, B., Dorna, C., Fretelliere, B., Garnier, I., Mangal, A., Neveu, F., Seillier, M., Soulie, R., \& Thomas, A. (2011). Le travail flexible à l'épreuve de la vie quotidienne : le cas du personnel soignant et éducatif en institution de santé. (Module interprofessionnel de santé publique). École des Hautes Études en Santé Publique.

Cheeseman, J. F., Webster, C. S., Pawley, M. D., Francis, M. A., Warman, G. R., \& Merry, A. F. (2011). Use of a new task-relevant test to assess the effects of shift work and drug abelling formats on anesthesia trainees' drug recognition and confirmation. Canadian Journal of Anesthesia, 58(1), 38-47.

Cheyrouze, M., Barthe, B., \& Barrau, H. (2016). 12-hour shifts: determining the role of temporal flexibility allowed by extended work periods to manage sleepiness variations of nurses and nursing work requirements. HEPS 2016, 5th edition of HEPS (Healthcare Ergonomics and Patient Safety) Conference - Healthcare and Society: new challenges, new opportunities. (Toulouse, 5-7 octobre).

Christol, J., Dorel, M., Quéinnec,, Y. \& Terssac, G. (de). (1979). Horaires de travail et régulation des conduits. Le travail Humain, 42(2), 211-229.

Décret $n^{\circ}$ 2010-1408 du 12 novembre 2010 relatif à la lutte contre les événements indésirables associés aux soins dans les établissements de santé. https://www.legifrance.gouv.fr/eli/ decret/2010/11/12/SASH1017107D/jo

Falzon, P. (2004). Ergonomie. Paris : Presses universitaires de France.

Falzon, P., Dicioccio, A., Mollo, V., \& Nascimento, A. (2013). Qualité réglée, qualité gérée. https:// halshs.archives-ouvertes.fr/halshs-00869623/document

Ferguson, S. A., Paech, G. M., Dorrian, J., Roach, G. D., \& Jay, S. M. (2011). Performance on a simple response time task: Is sleep or work more important for miners? Applied ergonomics, 42(2), 210-213.

Folkard, S., \& Åkerstedt, T. (1992). A three-process model of the regulation of alertnesssleepiness. In R. J. Broughton \& R. D. Ogilvie (Eds.) Sleep, Arousal, and Performance. 11-26. Boston: Birkhäuser.

Folkard, S., \& Tucker, P. (2003). Shift work, safety and productivity. Occupational Medicine, 53(2), 95-101.

Gadbois, C., \& Quéinnec, Y. (1984). Travail de nuit, rythmes circadiens et régulation des activités. Le Travail Humain, 47(3), 195-225.

Gaudart, C., \& Thébault, J. (2012). La place du care dans la transmission des savoirs professionnels entre anciens et nouveaux à l'hôpital. Industrial Relations, 67(2), 242-262.

Geiger-Brown, G. J., Rogers, V., Bausell, R., Trinkoff, A., Kane, R., \& Scharf, S. M. (2010). Lapses of attention and reaction time in sleep-deprived nurses working successive 12-hour shifts. Sleep, 33 (pp. A102-A103). SLEEP 2010, $24^{\text {th }}$ edition of SLEEP. Associated Professional Sleep Societies. (USA, June 8 2010).

Geiger-Brown, J., \& M Trinkoff, A. (2010) Is it time to pull the plug on 12-hour shifts? Journal of Nursing Administration, 40(9), 357-359

Geiger-Brown, J., Rogers, V. E., Trinkoff, A. M., Kane, R. L., Bausell, R. B., \& Scharf, S. M.

(2012). Sleep, sleepiness, fatigue, and performance of 12-hour-shift nurses. Chronobiology international, 29(2), 211-219. 
Girier, M. (2014). Résultats de l'enquête de l'ADRHESS sur les horaires en 12h. Les Rencontres RH de la santé, $3^{\text {ème }}$ édition (Montrouge, 26-27 septembre).

Gonon, O., Barthe, B., \& Gindro, G. (2009). Organisation du travail à l'hôpital : Comprendre l'articulation des temporalités pour agir sur la transformation des situations de travail. Actes du 44ème congrès de la SELF, 183-203. (Toulouse, 22-24 septembre).

Guérin, F., Laville, A., Daniellou, F., Duraffourg, J., \& Kerguelen, A. (1997). Comprendre le travail pour le transformer : la pratique de l'ergonomie. ANACT.

Institute of Medicine (2001). Crossing the quality chasm: A new health system for 21st century. Washington DC : National Academy Press.

Hoc, J. M. (2004). 31. La gestion de situation dynamique. In Falzon, P. Ergonomie. 515-530.

Presses Universitaires de France.

Kerguelen, A. (1995). Description et quantification en analyse ergonomique du travail : le cas de l'observation systématique. L'usage des méthodes statistiques dans l'étude du travail, 131-139.

Knauth, P. (2007). Extended work periods. Industrial health, 45(1), 125-136.

Lalandre, B. (2009). Les relations ambulancier-patient. In Appay, B. \& Jefferys, S. Restructurations, précarisations, valeurs. 379-38. Toulouse : Octarès.

Lancry, A. (2016). Chapitre IV - De nouvelles situations, de nouvelles façons de travailler. Que saisje ? 2, 69-83.

Leplat, J. (2000). L'analyse psychologique de l'activité en ergonomie. Toulouse : Octarès

Marias, D. J., II, J. H., Brillman, J. C., \& Tandberg, D. (1996). Effect of time of day and duration into shift on hazardous exposures to biological fluids. Academic Emergency Medicine, 3(6), 605-610.

Machi, M. S., Staum, M., Callaway, C. W., Moore, C., Jeong, K., Suyama, J., \& Hostler, D. (2012). The relationship between shift work, sleep, and cognition in career emergency physicians. Academic Emergency Medicine, 19(1), 85-91.

Manus, J.-M. (2002). L'infirmière et le travail de nuit : la vie à l'envers. Revue de l'infirmière, 81, $12-15$.

Matsumoto, K., \& Harada, M. (1994). The effect of night-time naps on recovery from fatigue following night work. Ergonomics, 37(5), 899-907.

Mollo, V., \& Falzon, P. (2004). Auto-and allo-confrontation as tools for reflective activities. Applied ergonomics, 35(6), 531-540.

Noulin, M. (2002). Ergonomie. Toulouse : Octarès.

Or, Z. \& Com-Ruelle, L. (2008). La qualité de soins en France : comment la mesurer pour l'améliorer ? Journal d'économie médicale, 26(6), 371-385.

Parent, P. A. (2008). L'évaluation des pratiques professionnelles, outil d'intégration de la démarche d'évolution continue de la qualité par les soignants. (Mémoire). École des Hautes Études en Santé Publique.

Perraut Soliveres, A. (2002). Le savoir de la nuit. Paris : PUF.

Prunier-Poulmaire, S. (2008). Concilier horaires et activité. Santé et travail, (61), 30-31.

Prunier-Poulmaire, S., \& Gadbois, C. (2005). Quand le questionnaire s'impose à l'ergonome. 
In Volkoff. L'ergonomie et les chiffres de la santé au travail. Ressources, tensions et pièges. Toulouse : Octarès, 75-86.

Quéinnec, Y., Teiger, C., \& Terssac, G. de. (2008). Repères pour négocier le travail posté. Toulouse : Octarès.

Scott, L. D., Rogers, A. E., Hwang, W. T., \& Zhang, Y. (2006). Effects of critical care nurses' work hours on vigilance and patients' safety. American Journal of Critical Care, 15(1), 30-37.

Simonet, P., Caroly, S., \& Clot, Y. (2011). Méthodes d'observation de l'activité de travail et prévention durable des TMS. Action et discussion interdisciplinaire entre clinique de l'activité et ergonomie. Activités, 8(8-1).

Smith, A.P., \& Wilson, M. (1990). The effects of naps during night duty on the performance and mood of female nurses working in intensive care unit. In Costa, G, Cesana, G., Kogi, K. \& Wedderburn, A. (Eds.) Shiftwork: Health, Sleep and Performance. 147-152. Frankfurt am Main : Peter Lang.

Terssac, G. (de)., Quéinnec, Y., \& Thon, B. (1983). Horaires de travail et organisation de l'activité de surveillance. Le Travail Humain, 46(1), 65-79.

Thayer, R. E. (1967). Measurement of activation through self-report. Psychological reports, 20(2), 663-678.

Tirilly, G., Barthe, B., \& Gentil, C. (2015). Pouvoir se reposer au cours du poste de nuit : un atout pour le travail ? Perspectives interdisciplinaires sur le travail et la santé, (17-2).

Toupin, C. (2008). Expérience et redéfinition de la tâche dans le travail des infirmières de nuit : Une recherche menée dans des unités de pneumologie. (Thèse de doctorat). Conservatoire National des Arts et Métiers.

Toupin, C. Barthe, B., \& Prunier-Poulmaire, S. (2013) Du temps contraint au temps construit : vers une organisation capacitante du travail de nuit, In P. Falzon Ergonomie Constructive (75-88), PUF : Paris.

Toupin, C., \& Volkoff, S. (2007). Experience and compromise in night shifts among hospital nurses. Ergonomia, 29(3-4).

Travail en 12 heures : les pièges à éviter pour les DRH. (2013). TecHopital.com : L'actualité de l'ingénierie hospitalière. Consulté le 12 janvier 2018 : http://www.techopital.com/Travail-en-12heures--les-pieges-a-eviter-pour-les-DRH-NS_1169.html

Van Daele, A., \& Carpinelli, F. (2001). La planification dans la gestion des environnements dynamiques : quelques apports récents de la psychologie ergonomique. Psychologie française, 46(2), 143-152.

Vermersch, P. (1991). L'entretien d'explicitation dans la formation expérientielle organisée. La formation expérientielle des adultes, 271-284.

Vincent, F. (2014). Travailler pour son « temps de repos» ? Sens et usages du temps hors travail des soignantes en 12 heures à l'hôpital public. Temporalités. Revue de sciences sociales et humaines, (20).

Vincent, F. (2017). Penser sa santé en travaillant en 12 heures. Les soignants de l'hôpital public entre acceptation et refus. Perspectives interdisciplinaires sur le travail et la santé, (19-1). 


\section{ANNEXES}

\section{Annexe 1 - Grille de l'entretien exploratoire}

Partie 1 : Le profil de l'infirmier (âge, expérience, horaires de travail)

Quel est votre âge?

Depuis combien de temps êtes-vous infirmier? Depuis combien de temps travaillez-vous en réanimation?

Etes-vous fixe de jour (avec 3 cycles de nuit) ou fixe nuit? Depuis combien de temps? Pour quelle(s) raison(s)?

Partie 2: La qualité des soins du point du point de vue de l'infirmier (critères, objectifs, situations problématiques, oublis)

À quoi vous fait penser l'expression « qualité du travail » ? Quels sont vos critères d'un « travail bien fait » et quels sont les objectifs que vous souhaitez atteindre dans votre travail?

Y-a-t-il des situations où ces critères et objectifs sont plus difficiles à atteindre ? Pouvezvous me décrire ces situations?

Dans votre travail, quelles sont les tâches qui peuvent être " presque oubliées » (que vous avez failli oublier) ? Selon vous, quels sont les « presque oublis » importants pour votre sécurité et celle des patients, et les « presque oublis » qui le sont moins?

Partie 3 : Les stratégies mises en œuvre par l'infirmier dans son travail

Dans les situations où les objectifs sont plus difficiles à atteindre, comment procédez-vous pour les atteindre malgré tout?

Comment gérez-vous les presque oublis ou les presque incidents (les incidents qui ont failli se produire) ? Comment parvenez-vous à les réduire?

Avez-vous repéré des contradictions entre les attentes et exigences de votre travail et la réalité du terrain? Si oui, pouvez-vous me les expliquer?

Partie 4 : La somnolence de l'infirmier (variations, incidence sur le travail, stratégies associées)

Au cours des 12 heures du poste de jour/nuit, y a-t-il des périodes où vous ressentez une augmentation de votre somnolence? Si oui, lesquelles?

Cette augmentation de votre somnolence a-t-elle une incidence sur les résultats de votre travail?

Comment gérez-vous l'augmentation de votre somnolence?

Partie 5 : L'incidence de la durée du poste et de l'enchainement des postes sur le travail. Selon vous, est-ce que la durée de votre poste de jour/nuit - c'est-à-dire 12 heures - a une incidence sur la qualité des soins telle que vous venez de l'aborder? 
Selon vous, est-ce que le fait de travailler plusieurs jours ou plusieurs nuits d'affilée a une incidence sur la qualité des soins telle que vous venez de l'aborder?

\section{Annexe 2 - Echelle de Thayer (1967)}

Chaque mot de la liste correspond à un sentiment ou une humeur. Utilisez l'échelle d'évaluation suivante pour décrire chacun d'entre eux dans votre cas et en ce momentmême.

++ Je me sens très

+ Je me sens un peu

? Je ne sais pas

0 Je ne me sens pas du tout

Entourez rapidement votre réponse, sans oublier de mot et en vous fiant à votre première impression. Le questionnaire devrait vous prendre une minute ou deux.

\begin{tabular}{|l|l|}
\hline Sentiment/humeur & Échelle d'évaluation \\
\hline Actif (ve) & $+++? \quad 0$ \\
\hline Indifférent (e) & $+++? 0$ \\
\hline Endormi (e) & $+++? 0$ \\
\hline Mal à l'aise & $+++? 0$ \\
\hline Energique & $+++? 0$ \\
\hline Calme & $+++? 0$ \\
\hline Fatigué (e) & $+++? 0$ \\
\hline Dynamique & $+++? 0$ \\
\hline Détendu (e) & $+++? 0$ \\
\hline Attentif (ve) & $+++? 0$ \\
\hline Somnolent (e) & $+++? 0$ \\
\hline Inquiet (e) & $+++? 0$ \\
\hline « La pêche» & $+++? 0$ \\
\hline Tranquille & $+++? 0$ \\
\hline Eveillé (e) & $+++? 0$ \\
\hline Enervé (e) & $+++? 0$ \\
\hline
\end{tabular}




\begin{tabular}{|l|l|}
\hline Silencieux (se) & $+++? 0$ \\
\hline En forme & $+++? 0$ \\
\hline Tendu (e) & $+++? 0$ \\
\hline Crispé (e) & $+++? 0$ \\
\hline
\end{tabular}

\section{Annexe 3 - Echelle d'auto-évaluation de la qualité des soins}

Dans ce questionnaire, les questions correspondent aux critères d'un « travail bien fait ». Lisez-attentivement chacune d'elle et évaluez-vous de façon spontanée. Les modalités de réponse vont de la couleur verte (évaluation positive) à la couleur rouge (évaluation négative).

Cochez rapidement la couleur qui vous convient en fonction de ce qui s'est passé depuis le dernier questionnaire, sans oublier de ligne et en vous fiant à votre première impression.

\begin{tabular}{|l|l|l|l|l|}
\hline & & & & \\
\hline $\begin{array}{l}\text { 1) Avez-vous accompli toutes les tâches } \\
\text { que vous vous étiez fixées ? }\end{array}$ & & & & \\
\hline $\begin{array}{l}\text { 2) Avez-vous respecté les règles } \\
\text { d'hygiène et de sécurité dans } \\
\text { 1'administration des soins, l'utilisation } \\
\text { du matériel et la tenue des locaux? }\end{array}$ & & & & \\
\hline $\begin{array}{l}\text { 3) Avez-vous travaillé avec un matériel } \\
\text { adéquat et fonctionnel? }\end{array}$ & & & & \\
\hline $\begin{array}{l}\text { 4) Avez-vous assuré une prise en charge } \\
\text { "globale» de vos patients? }\end{array}$ & & & & \\
\hline $\begin{array}{l}\text { 5) Avez-vous été satisfait(e) de } \\
\text { l'ambiance, l'entraide et la } \\
\text { communication avec vos collègues? }\end{array}$ & & & & \\
\hline $\begin{array}{l}\text { 6) Avez-vous fait rapidement et } \\
\text { correctement le lien entre les } \\
\text { prescriptions et l'état de vos patients? }\end{array}$ & & & & \\
\hline $\begin{array}{l}\text { 7) Avez-vous su vous organiser dans } \\
\text { votre travail? }\end{array}$ & & & & \\
\hline $\begin{array}{l}\text { 8) Avez-vous su anticiper et/ou gérer les } \\
\text { imprévus? }\end{array}$ & & & & \\
\hline $\begin{array}{l}\text { 9) Êtes-vous resté(e) concentré(e)? } \\
\text { 10) Avez-vous été certain(e) de n'avoir } \\
\text { rien oublié ? }\end{array}$ & & & & \\
\hline $\begin{array}{l}\text { 11) Avez-vous été certain(e) de ne pas } \\
\text { vous être trompé(e)? }\end{array}$ & & & \\
\hline
\end{tabular}

\section{Annexe 4 - Questionnaire des régulations}

Voici une liste de stratégies qui ont pu vous permettre d'effectuer votre travail.

Merci de cocher celle(s) que vous avez utilisée(s) au cours des dernières heures.

Accélérer sa cadence de travail 
Ralentir sa cadence de travail

Fonctionner par priorités

S’avancer dans son travail

Vérifier plusieurs fois la feuille de réanimation

Support écrit personnel

Organisation mentale

Faire appel au(x) collègue(s)

Repousser ou réduire son temps de pause

Autre(s)

Commentaires :

Voici une liste de stratégies qui ont pu vous permettre de gérer votre état d'éveil.

Merci de cocher celle(s) que vous avez utilisée(s) au cours des dernières heures.

Trouver des occupations

Prendre une pause à l'extérieur du module

Se reposer dans le module

Commentaires :

\section{RÉSUMÉS}

Les 2 x 12 se déploient massivement en milieu hospitalier mais ne sont pas sans risques pour la qualité des soins. Pour à la fois gérer les variations de leur vigilance et atteindre leurs objectifs de soin, les infirmiers emploient dans leur activité des stratégies de régulation. Il s'agit d'étudier la qualité des soins du point de vue des infirmiers et de comprendre en quoi leurs stratégies peuvent contribuer à sa stabilité. L'étude porte sur le travail des infirmiers d'un service de réanimation médicale en poste de nuit de 12 heures. Une observation globale de 18 infirmiers est d'abord menée. Douze infirmiers sont ensuite interrogés en entretiens exploratoires sur leur conception de la qualité des soins et leurs stratégies de régulation. De ces entretiens sont construits trois questionnaires. À quatre moments du poste de nuit, 24 infirmiers évaluent leur vigilance, la qualité des soins et indiquent leurs stratégies de régulation. Ils sont également observés en continu. Après chacune des quatre passations, nous les invitons à commenter leurs réponses en entretien individuel. D'après les résultats, les infirmiers s'estiment de moins en moins vigilants au fil du poste alors que leur perception de la qualité des soins reste très positive. C'est grâce à leur «scénario de travail " élaboré en début de nuit qu'ils la maintiennent à un niveau satisfaisant. Ce scénario leur permet d'anticiper les imprévus, de fiabiliser les soins et d'aménager des temps de repos. 
The $2 \times 12$ is massively deployed in hospital settings but is not without risks for the quality of care. To take into account their vigilance and the care they must give, the nurses use regulation strategies in their activity. This essentially means studying the quality of care from the nurses' point of view and looking to understand how regulating their activity might contribute to its stability. The study concerns solely the work of nurses on 12-hour night shifts in a medical reanimation. First, 18 nurses are observed. In exploratory interviews, 12 nurses are then questioned about their personal conceptions of the quality of care given and their regulation strategies. From these talks, we established three questionnaires. At four different times throughout their night shifts, nurses are asked to evaluate their vigilance, the quality of care given and to indicate their regulation strategies. They are also continually observed. After every test period, we asked them to comment on their answers in an individual interview. The results show that the nurses feel less and less vigilant as the night goes on, although their view on the quality of care given remains positive at all times. It is their "work scenario", developed early in the night that enables them to maintain a satisfactory level of quality. This scenario allows them to enhance the reliability of their care, increase their work load and organise break times.

\section{INDEX}

Mots-clés : hôpital, travail de nuit, régulation, qualité des soins, ergonomie

\section{AUTEURS}

\section{MARLÈNE CHEYROUZE}

Université Toulouse Jean Jaurès, Laboratoire CLLE-LTC, UMR 5263 CNRS, Maison de la Recherche, 5 allée Antonio Machado 31058 Toulouse Cedex 9 marlene.cheyrouze@univ-tlse2.fr

\section{BÉATRICE BARTHE}

Université Toulouse Jean Jaurès, UFR de Psychologie, Laboratoire CLLE-LTC, UMR 5263 CNRS, Maison de la Recherche, 5 allée Antonio Machado 31058 Toulouse Cedex 9 beatrice.barthe@univ-tlse2.fr 